\title{
Rapamycin reversal of VEGF-C-driven lymphatic anomalies in the respiratory tract
}

\author{
Peter Baluk, ${ }^{1}$ Li-Chin Yao, ${ }^{1}$ Julio C. Flores, ${ }^{1}$ Dongwon Choi, ${ }^{2}$ Young-Kwon Hong, ${ }^{2}$ \\ and Donald M. McDonald' \\ 'Cardiovascular Research Institute, Department of Anatomy, and Helen Diller Family Comprehensive Cancer Center, \\ University of California, San Francisco, San Francisco, California, USA. ${ }^{2}$ Norris Comprehensive Cancer Center, University of \\ Southern California, Los Angeles, California, USA.
}

\begin{abstract}
Lymphatic malformations are serious but poorly understood conditions that present therapeutic challenges. The goal of this study was to compare strategies for inducing regression of abnormal lymphatics and explore underlying mechanisms. CCSP-rtTA/tetO-VEGF-C mice, in which doxycycline regulates VEGF-C expression in the airway epithelium, were used as a model of pulmonary lymphangiectasia. After doxycycline was stopped, VECF-C expression returned to normal, but lymphangiectasia persisted for at least 9 months. Inhibition of VEGFR-2/VEGFR-3 signaling, Notch, $\beta$-adrenergic receptors, or autophagy and antiinflammatory steroids had no noticeable effect on the amount or severity of lymphangiectasia. However, rapamycin inhibition of $m$ TOR reduced lymphangiectasia by $76 \%$ within 7 days without affecting normal lymphatics. Efficacy of rapamycin was not increased by coadministration with the other agents. In prevention trials, rapamycin suppressed VECF-C-driven mTOR phosphorylation and lymphatic endothelial cell sprouting and proliferation. However, in reversal trials, no lymphatic endothelial cell proliferation was present to block in established lymphangiectasia, and rapamycin did not increase caspasedependent apoptosis. However, rapamycin potently suppressed Prox1 and VEGFR-3. These experiments revealed that lymphangiectasia is remarkably resistant to regression but is responsive to rapamycin, which rapidly reduces and normalizes the abnormal lymphatics without affecting normal lymphatics.
\end{abstract}

Authorship note: P. Baluk and L.C. Yao contributed equally to this work.

Conflict of interest: The authors have declared that no conflict of interest exists.

Submitted: August 11, 2016

Accepted: July 6, 2017

Published: August 17, 2017

Reference information:

JCI Insight. 2017;2(16):e90103.

https://doi.org/10.1172/jici.

insight.90103.

\section{Introduction}

Lymphatic malformations, including generalized lymphatic anomalies, Gorham-Stout disease, Kaposiform lymphangiomatosis, and related lymphatic growths defined by the International Society for the Study of Vascular Anomalies classification (1), are uncommon but serious pathological conditions that present diagnostic and therapeutic challenges (2-4). Pulmonary lymphangiectasia, in which airway and lung lymphatics are abnormally expanded and leaky (5-7), is a feature of many widespread lymphatic anomalies. After a long history of empirical management of these disorders, progress is underway. Discovery of mutations in RAS signaling or PIK3CA in some lymphatic malformations (8-10) provides insight into potential mechanisms. The mTOR inhibitor rapamycin (sirolimus) has yielded promising results in the treatment of patients with lymphatic malformations $(3,4,11,12)$. However, the absence of experimental models for examining pathophysiology and testing innovative treatments is a limitation.

The goal of the present study was to identify strategies for treating lymphatic anomalies in a mouse model, in which mechanistic insight could be obtained and translated to humans. We used transgenic mice to compare the efficacy of prevention and reversal of lymphatic anomalies and explore underlying mechanisms. We tested the hypothesis that lymphatic anomalies can be prevented or reversed by blocking pathways that are essential for the growth or survival of lymphatic endothelial cells. For this purpose, we used inducible CCSP/ VEGF-C-transgenic mice that develop lymphatic anomalies similar to congenital pulmonary lymphangiectasia (6). After activation of VEGF-C overexpression in airway epithelial cells by administration of doxycycline, lymphangiectasia rapidly develops in the airways and lungs. Strikingly, the lymphangiectasia does not regress spontaneously, not even over the 19 months after VEGF-C expression returns to normal (6). 
After determining the optimal age, the amount and duration of doxycycline, and the washout period after treatment to allow VEGF-C expression to return to normal, we compared the efficacy of candidate treatment strategies for reversing lymphatic anomalies in CCSP/VEGF-C mice. Inhibition of VEGFR-2 and VEGFR-3 by function-blocking antibodies $(6,13)$ was compared with inhibition of Notch signaling by the $\gamma$-secretase inhibitor DAPT (14), suppression of $\beta$-adrenergic receptor signaling by propranolol ( 15 , $16)$, inhibition of autophagy by chloroquine $(17,18)$, suppression of inflammation by dexamethasone $(13$, $19)$, or inhibition of mTOR by rapamycin $(18,20)$. Surprisingly, only rapamycin led to significant reversal of lymphangiectasia in this model, and the efficacy of rapamycin was not improved by coadministration of anti-VEGFR-2/VEGFR-3 antibodies, propranolol, or chloroquine.

We therefore sought to determine the dose response and time course of effects of rapamycin on lymphangiectasia in this model. Because significant reversal of lymphangiectasia was found only with the highest dose of rapamycin (20 mg/kg daily), we assessed rapamycin blood levels, systemic effects, and potential toxicity. Based on evidence that VEGF-C-driven lymphangiogenesis in CCSP/VEGF-C mice can be readily prevented by inhibition of VEGFR-2/VEGFR-3 signaling (6), we examined effects of rapamycin on lymphatics at baseline, during growth, and in a prevention model before exploring the mechanism of rapamycin reversal of established lymphangiectasia. Cellular mechanisms underlying the actions of rapamycin on lymphatic endothelial cells in these mice were assessed by examining changes in mTOR signaling, suppression of lymphatic sprouting and proliferation, induction of apoptosis, and changes in Prox1 and VEGFR-3.

The studies revealed that markers of mTOR signaling rapidly increased during VEGF-C-driven lymphatic growth and that rapamycin prevented this increase and lymphatic sprouting and proliferation in vivo. However, once lymphangiectasia was established and VEGF-C expression returned to normal, there was little lymphatic endothelial cell proliferation to suppress, and rapamycin did not induce detectable caspase-dependent apoptosis in the abnormal lymphatics. Yet, rapamycin did reduce Prox1 and VEGFR-3 mRNA and protein in CCSP/VEGF-C mice. The studies confirmed the resilience of abnormal lymphatics against regression and revealed that rapamycin reduced the severity of lymphatic anomalies in this model but only at doses higher than those producing many other effects.

\section{Results}

\section{CCSP/VEGF-C mice as a model for studying reversal of pulmonary lymphangiectasia}

Newborn CCSP/VEGF-C mice develop widespread lymphangiectasia in the airways and lungs after doxycycline activation of VEGF-C overexpression (6). The severity of lymphangiectasia depends on age and amount and duration of doxycycline exposure (6). Neonatal mice develop more severe lymphangiectasia than older mice (6).

In evaluating the suitability of CCSP/VEGF-C mice for studies of lymphatic regression, we used neonates in initial experiments to confirm the rapid increase in VEGF-C expression and lymphangiogenesis after doxycycline exposure and the persistence of abnormal lymphatics after doxycycline withdrawal, as shown previously (6). Tracheas of neonates given doxycycline from P0 to P7 $(0.1 \mathrm{mg} / \mathrm{ml}$ in maternal drinking water $)$ had 23-fold of the normal VEGF-C expression, and the mucosa contained confluent, sheet-like lymphatics (lymphangiectasia) (Supplemental Figure 1, A-D; supplemental material available online with this article; https://doi.org/10.1172/jci.insight.90103DS1). VEGF-C mRNA expression and lymphatic density were little changed 7 days after doxycycline was withdrawn (Supplemental Figure 1, A and B). Neonates given onetenth the doxycycline concentration $(0.01 \mathrm{mg} / \mathrm{ml})$ from P0 to P7 had lower VEGF-C mRNA expression (3-2fold of normal) and less lymphangiectasia, but VEGF-C expression was still 2-fold of normal at 7 days after doxycycline was stopped (Supplemental Figure 1, E and F). The sustained elevation of VEGF-C expression complicated the use of neonatal mice for studying the regression of lymphatic anomalies.

To circumvent this problem, we assessed the development of lymphangiectasia in weanling mice that were given doxycycline $(0.01 \mathrm{mg} / \mathrm{ml}$ in drinking water) from P21 to P28. This approach also eliminated the confounding variable of giving doxycycline through the mother's drinking water. CCSP/VEGF-C mice on doxycycline from P21 to P28 had $100 \%$ survival $(n=372)$, in contrast to appreciable mortality from chylothorax in neonates (6). After doxycycline for 7 days (P28), VEGF-C expression in the trachea averaged 13-fold of the baseline value (Figure 1A), and the number of Prox1-positive lymphatic endothelial cells was 4-fold of the baseline number (Figure 1B). Sprouting lymphatics covered most cartilage rings (Figure 1, C 
Lymphangiectasia in trachea of CCSPNEGF-C mice: Control, doxycycline 7d, and off doxycycline 14d

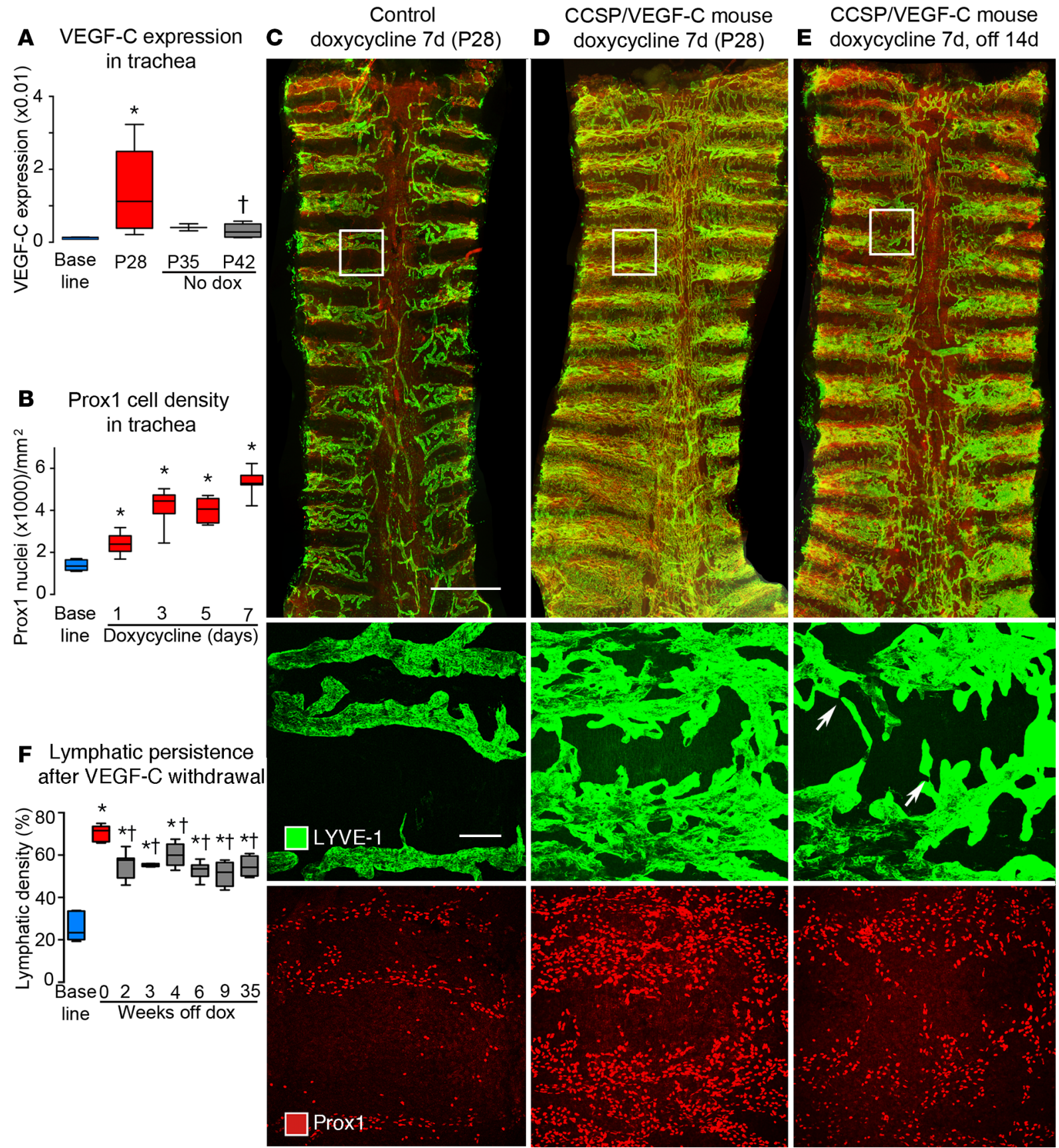

Figure 1. Lymphatics in tracheas of CCSP/VEGF-C mice before and after doxycycline. (A) VEGF-C mRNA expression (relative to $\beta$-actin) in trachea increased on doxycycline and returned almost to baseline after 14-day doxycycline washout period. (B) Number of Prox1-positive nuclei before and during doxycycline administration. (C-E) Overview of tracheal whole mounts stained for LYVE-1 (green) and Prox1 (red) from mice given $0.01 \mathrm{mg} / \mathrm{ml}$ doxycycline for 7 days (P21-P28) and perfused at P28 (C and D) or after 14-day washout period (P42) (E). (C) Trachea from a control (single-transgenic) mouse, with normal lymphatics restricted to intercartilage spaces and over the trachealis muscle at the center. (D) Trachea from a double-transgenic CCSP/VECF-C mouse after doxycycline for 7 days, where lymphatics cover a much larger area and increase in abundance toward the caudal end. (E) Trachea from a P42 double-transgenic mouse 14 days after doxycycline withdrawal; lymphatics are less numerous than at P28, especially in central muscular region, but most of the lymphatic abnormality persists. Boxed regions are shown at higher magnification below. The middle row shows the boxed region stained for LYVE-1 showing normal lymphatics (C), lymphangiectasia at P28 (D), and spontaneous regression between P28 and P42 (E). Some lymphatics are narrowed regions (arrows). The lower row shows Prox1-positive nuclei at the beginning (C) and end of doxycycline treatment (D) and 14 days after withdrawal (E). Scale bar: 1 millimeter (top row); $50 \mu \mathrm{m}$ (middle and bottom rows). (F) Extent of LYVE-1 lymphatics in trachea before doxycycline (blue box), after doxycycline (red box), and after doxycycline withdrawal (gray boxes). Values show the lymphatic expansion during doxycycline and some regression during the 2-week withdrawal period (P28-P42) but none thereafter throughout the 9-month study period (gray boxes were not significantly different from each other). $n=3$ to 10 mice/group. ${ }^{*} P<0.05$ vs. baseline group, ${ }^{\dagger} P<0.05$ vs. P28, ANOVA. Box and whisker plots show the median, first and third quartiles, and maximum and minimum. 
A Standard protocol for studying reversal of lymphangiectasia in CCSP/VEGF-C mice

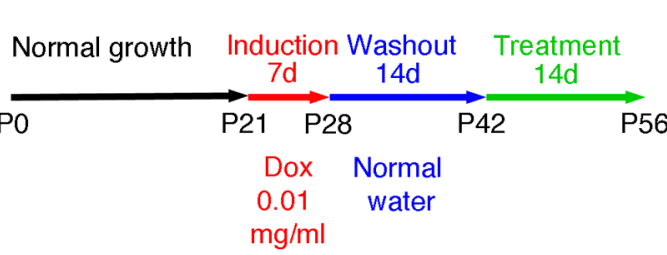
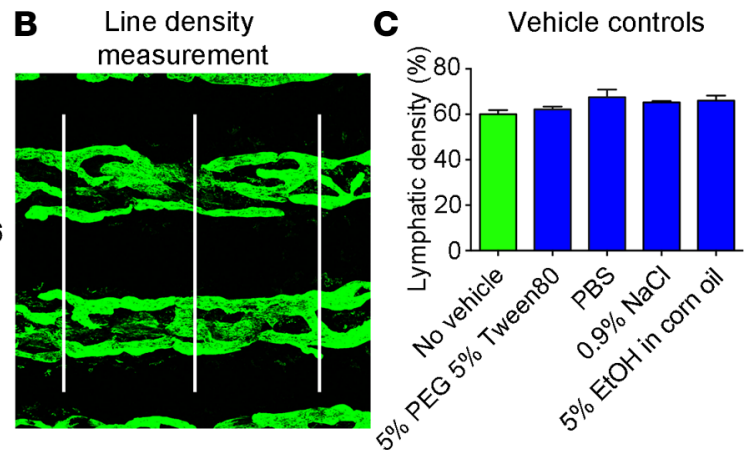

Efficacy of 6 treatments for reversing lymphangiectasia in CCSP/VEGF-C mice

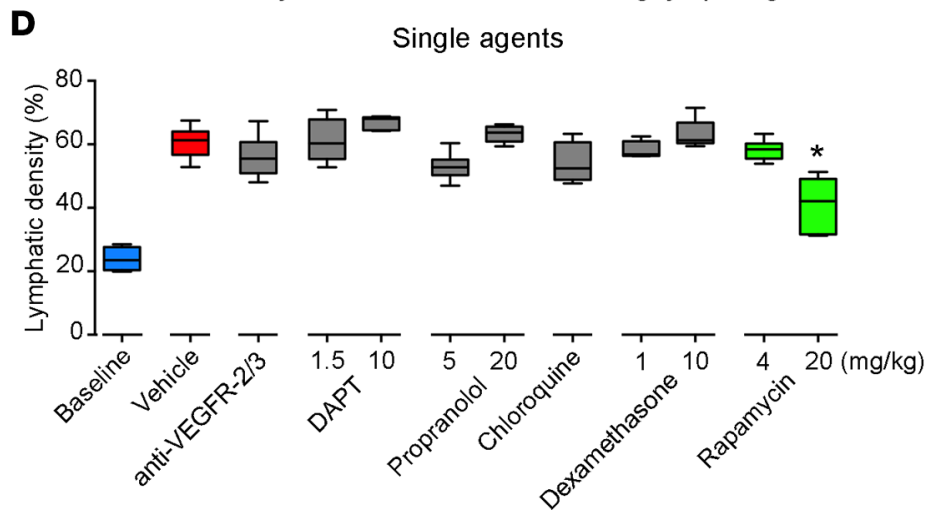

Figure 2. Standard protocol and comparison of effects of six treatment strategies. (A) Standard protocol for studying effects of treatment on lymphangiectasia in CCSP/VEGF-C mice on doxycycline $(0.01 \mathrm{mg} / \mathrm{ml}$ ) for 7 days, off doxycycline for 14 days, and then treated for 14 days ( $n=4-6 \mathrm{mice} / \mathrm{group}$ ). (B) Approach for measuring line density of tracheal lymphatics stained for LYVE-1 by overlaying 3 parallel lines and assessing the fraction of pixels with a brightness of 100 or greater (on a scale of 0-255) with Image). (C) Density of tracheal lymphatics in CCSP/VECF-C mice (P42-P56) after treatment with one of several vehicles used for the active therapeutics. (D) Lymphatic density in tracheas of CCSP/VEGF-C mice treated in the standard protocol with doxycycline (P21-P28), off doxycycline (P28-P42), and treated with one of six therapeutics for 14 days (P42-P56). Only the high dose of rapamycin (green box, $20 \mathrm{mg} / \mathrm{kg}$ ) induced significant regression compared with the vehicle-treated group (red box). Treatment with lower doses of candidate therapeutics had no significant effect. (E) Effects of treatment with rapamycin in combination with other agents (P42-P56). The amount of lymphatic regression was not significantly greater $(\dagger)$ than that found after rapamycin alone (green box) but was less than that after treatment with vehicle ${ }^{*} P<0.05$ vs. vehicle, ANOVA). Box and whisker plots show the median, first and third quartiles, and maximum and minimum.

and D, and Supplemental Figure 1, G and H). Sprouts had uniformly strong staining for neuropilin-2, VEGFR-2, and VEGFR-3 but weaker staining for LYVE-1 (Supplemental Figure 2, A-C). All of these markers had strong staining in nonsprouting regions.

Some spontaneous regression of lymphangiectasia occurred during the 14-day washout period after doxycycline was stopped (from P28 to P42) (Figure 1E), but no further reduction was found from P42 to the end of the 35-week study period (Figure 1F). Expression of VEGF-C mRNA was 73\% lower after doxycycline was withdrawn for 7 days (P35) and 79\% lower after withdrawal for 14 days (P42, Figure 1A). Unlike the prominent lymphangiogenesis in the trachea, lymphatics appeared normal in ear skin and diaphragm of these mice (Supplemental Figure 3, A-D).

\section{Contrasting efficacy of six treatment strategies}

From studies of weanling CCSP/VEGF-C mice, we adopted a standard protocol (Figure 2A) that had the attributes of inducing uniform lymphangiectasia, accompanied by consistent survival, limited spontaneous regression, and rapid decrease in VEGF-C mRNA after withdrawal of doxycycline. The efficacy of agents was tested for reversal of lymphangiectasia in these mice by giving doxycycline $(0.01 \mathrm{mg} / \mathrm{ml})$ in drinking water for 7 days (age P21-P28), replacing doxycycline with plain water (washout period) for 14 days (age $\mathrm{P} 28-\mathrm{P} 42$ ), and treating with the agent for 14 days (age P42-P56). The extent of mucosal lymphatics was measured in tracheal whole mounts at P56 (Figure 2B).

Six strategies were tested based on reports of efficacy for lymphatic anomalies, hemangiomas, or related conditions: (a) function-blocking antibodies for VEGFR-2 (DC101) and VEGFR-3 (mF4-31C1) (40 mg/kg 
of each i.p., alternate days) (6); (b) Notch signaling inhibitor DAPT (1.5 or $10 \mathrm{mg} / \mathrm{kg}$ gavage, 5 days/week) (21, 22); (c) pan $\beta$-receptor blocker propranolol (5 or $20 \mathrm{mg} / \mathrm{kg}$ i.p. daily) (23-25); (d) autophagy inhibitor chloroquine $(50 \mathrm{mg} / \mathrm{kg}$ i.p. daily) $(18,26)$; (e) antiinflammatory steroid dexamethasone (1 or $10 \mathrm{mg} / \mathrm{kg}$ i.p. daily) $(13,19)$, or (f) mTOR inhibitor rapamycin $(0.5$ to $20 \mathrm{mg} / \mathrm{kg}$ i.p. daily) $(27,28)$. None of the vehicles used for these agents had a measurable effect on amount of lymphangiectasia in this model (Figure 2C).

Treatment of CCSP/VEGF-C mice for 14 days with VEGFR-2/3-blocking antibodies, DAPT, propranolol, chloroquine, or dexamethasone did not significantly reduce the severity of lymphangiectasia assessed by the extent of LYVE-1-positive lymphatics in the tracheal mucosa (Figure 2D). In addition, no significant differences were found between the doses used (Figure 2D). Of these approaches, rapamycin $(20 \mathrm{mg} / \mathrm{kg}$ ) stood out as the only agent that significantly reduced lymphangiectasia in this model (Figure 2D). A lower dose of rapamycin ( $4 \mathrm{mg} / \mathrm{kg}$ ) had no significant effect (Figure 2D). Rapamycin did not have a larger effect on lymphangiectasia when given in combination with VEGFR-2/3-blocking antibodies, propranolol, or chloroquine for 14 days (Figure 2E).

\section{Dose response of rapamycin}

Rapamycin has shown efficacy in clinical trials of lymphangiomatosis and other lymphatic anomalies $(3,4$, $11,12)$. Rapamycin has also been used extensively in mice over a wide range of doses $(29,30)$. To determine the relationship between the dose of rapamycin and the extent of reversal of lymphangiectasia in CCSP/ VEGF-C mice, we compared doses from 0.5 to $20 \mathrm{mg} / \mathrm{kg}$ i.p. daily for 14 days. The extent of LYVE-1stained lymphatics in tracheas had a linear relationship with dose $\left(\mathrm{R}^{2}=0.7, P<0.0001\right)$, but the reduction in lymphangiectasia was significant only at the highest dose of rapamycin (20 mg/kg) (Figure 3A). Blood levels of rapamycin in the same mice were measured at 1 hour and 24 hours after the last dose (Figure 3, B and C). The overall half-life calculated from these values was 6.5 hours, with average half-lives ranging from 6.0 hours for the $0.5 \mathrm{mg} / \mathrm{kg}$ dose to 7.5 hours for the $20 \mathrm{mg} / \mathrm{kg}$ dose. Average rapamycin concentrations in serum at 1 hour ranged from 800 to $7,000 \mathrm{ng} / \mathrm{ml}$ according to the dose (Figure 3B). At 24 hours, the concentration was less than $200 \mathrm{ng} / \mathrm{ml}$ after doses of $5 \mathrm{mg} / \mathrm{kg}$ or less but was $800 \mathrm{ng} / \mathrm{ml}$ after the $20 \mathrm{mg} / \mathrm{kg}$ dose (Figure $3 \mathrm{C}$ ).

\section{Systemic effects and potential toxicity of rapamycin}

The high dose of rapamycin required to reduce lymphangiectasia in CCSP/VEGF-C mice led to an assessment of systemic effects and possible toxicity. CCSP/VEGF-C mice given rapamycin $20 \mathrm{mg} / \mathrm{kg}$ daily for 14 days had $100 \%$ survival $(n=66)$. Body weight was affected in a dose-dependent manner (Figure 3D), as reported previously (31). Body weight gain slowed in mice receiving the lowest dose of rapamycin $(0.5$ $\mathrm{mg} / \mathrm{kg}$ ) and decreased $4 \%$ in mice receiving the highest dose $(20 \mathrm{mg} / \mathrm{kg})$ for 14 days (Figure 3D). Weight of the thymus (Figure 3E) and blood lymphocyte count (Figure 3F) were also significant reduced, as found by others $(32,33)$. Total blood leukocytes, erythrocytes, hematocrit, and platelets were not significantly changed at even the highest dose (Figure 3, G-J).

\section{Rapamycin time course of reversal of Iymphangiectasia}

In untreated CCSP/VEGF-C mice, LYVE-1-positive lymphatics formed dense networks between cartilage rings and partially covered the rings (Figure 4A), where they were normally absent (Figure 1C). By comparison, lymphangiectasia was substantially reduced after rapamycin treatment for 7 days. The greatest reduction was over cartilage rings (Figure 4B). Changes in some lymphatics were evident after 1 day of rapamycin, when regions of vessel narrowing (Figure $4 \mathrm{C}$, arrows) and loss of the luminal membrane protein podocalyxin were found (Figure 4D). Some lymphatics had a more normal tubular appearance (Figure $4 \mathrm{~B}$, arrows). The reduction in lymphangiectasia was maximal at 7 days and was about the same at 14 days (Figure 4E and Supplemental Figure 3E).

The number of lymphatic endothelial cells was estimated by counting Prox1-positive cell nuclei to determine whether the reduction in lymphangiectasia reflected a decrease in cell size or cell number. This approach revealed that the tracheas of CCSP/VEGF-C mice on rapamycin for 7 days had fewer Prox1-positive nuclei (Figure 4, F and G). Counts revealed 57\% fewer Prox1-positive nuclei at 2 days (Figure 4H and Supplemental Figure $3 \mathrm{~F})$. The largest decrease $(89 \%)$ was found at 14 days (Figure $4 \mathrm{H})$. The reduction in Prox1-positive nuclei after rapamycin treatment was evident before the decrease in the extent of LYVE-1 staining (Figure $4 \mathrm{E})$. One reason was that Prox1 marked lymphatic endothelial cell nuclei present throughout the thickness of the mucosa, while LYVE-1 staining showed the 2-dimensional extent of lymphatics over the tracheal surface. 

A
Rapamycin dose-response
B
$1 \mathrm{hr}$ after last injection
C
$24 \mathrm{hr}$ after last injection

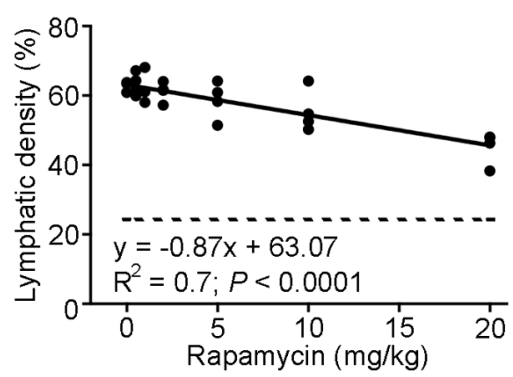

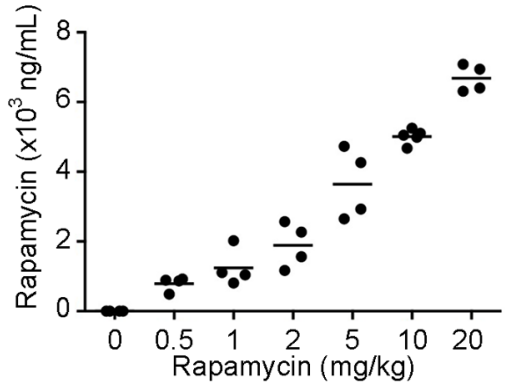

Rapamycin ( $\mathrm{mg} / \mathrm{kg})$

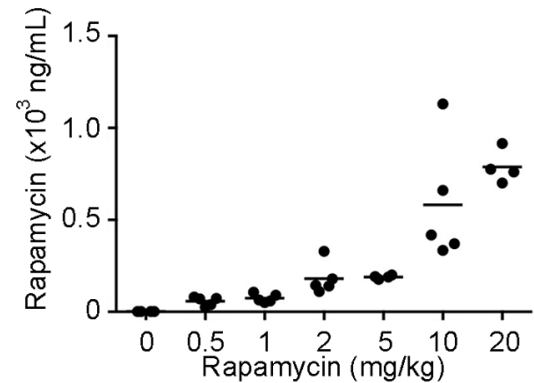

$\mathbf{F}$

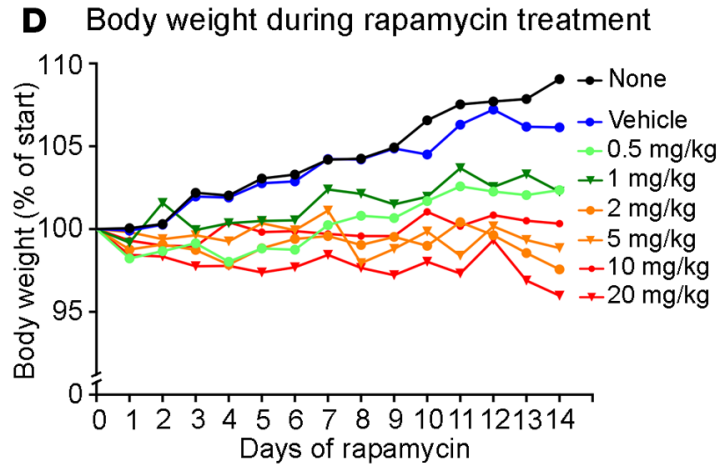

E

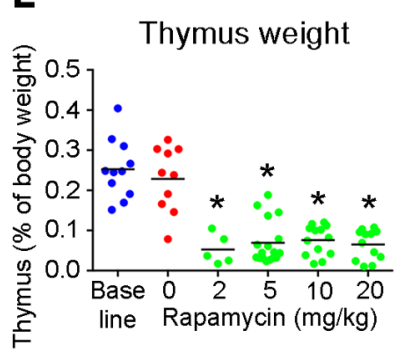

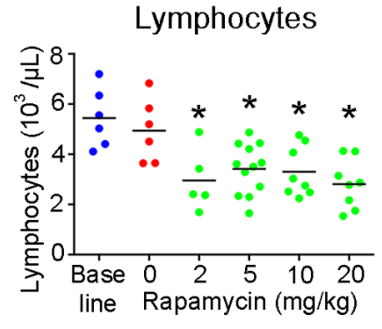
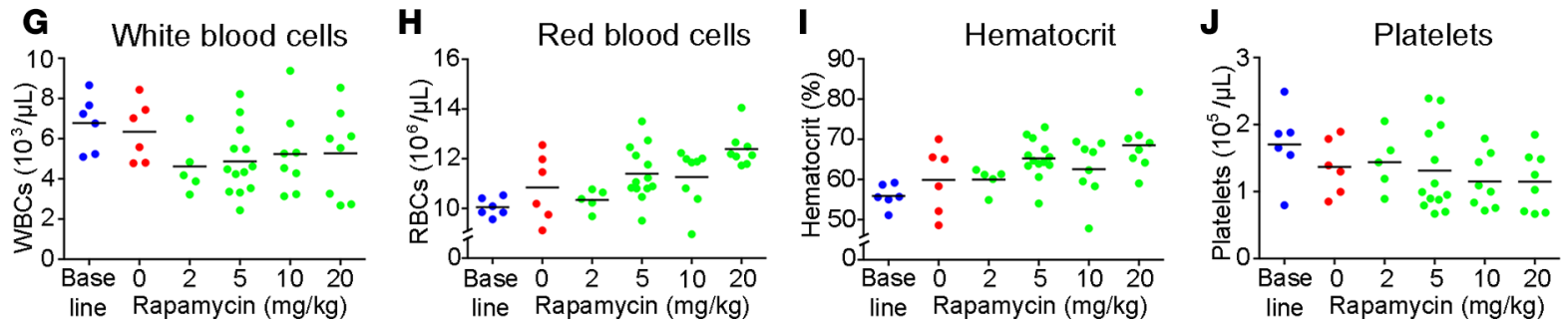

Figure 3. Dose response and assessment of toxicity of rapamycin. Dose-response effect of rapamycin (0.5-20 mg/kg i.p. daily, P42-P56) in CCSP/VEGF-C mice for 14 days on reversal of lymphangiectasia (A). Dashed line in $\mathbf{A}$ marks the value for tracheal lymphatics in normal mice. Rapamycin blood level at 1 hour (B) and 24 hours (C) after last dose. Body weight (D), thymus weight (E), and blood counts for lymphocytes (F), white blood cells (C), red blood cells (H), hematocrit (I), and platelets (J) at 24 hours after final dose. The baseline value represents that for wild-type mice. ${ }^{*} P<0.05$ vs. vehicle, ANOVA.

Because of these different properties, the two values reflected separate features, and an even more informative indicator of the effect of rapamycin on lymphangiectasia was the product of the two indices: numerical density of Prox1-positive cell nuclei in the mucosa times the surface density of LYVE-1 staining (Figure 4I). This calculation provided an estimate of the number of lymphatic endothelial cells per cubic millimeter of tracheal mucosa ( $80-\mu \mathrm{m}$ thickness) scaled to the extent of LYVE-1 staining. Accordingly, the number of lymphatic endothelial cells in regions of lymphangiectasia was reduced by $76 \%$ in mice treated with rapamycin for 7 days and by the same amount in mice treated for 14 days (Figure $4 \mathrm{I}$ ).

\section{Prevention of VEGF-C-driven lymphangiectasia by rapamycin}

VEGF-C-driven lymphangiogenesis in CCSP/VEGF-C mice is rapidly promoted by doxycycline and can be readily prevented by inhibition of VEGFR-2/VEGFR-3 signaling; however, it is much more difficult to reverse (6). Because of this background, we examined changes in mTOR signaling and other effects of rapamycin on lymphatics at baseline, during growth, and in a prevention model before using the same readouts to assess reversal. Lymphatic sprouting and growth were studied after doxycycline for 2 to 7 days. In the prevention trial, rapamycin was given throughout the period of doxycycline-induced VEGF-C production (age P21-P28). Cellular mechanisms underlying the actions of rapamycin on lymphatic endothelial cells in vivo were assessed by examining changes in mTOR signaling, suppression of sprouting and proliferation, induction of apoptosis, and changes in Prox1 and VEGFR-3 immunofluorescence. 
A LYVE-1 lymphatics vehicle $7 d$ (P49)

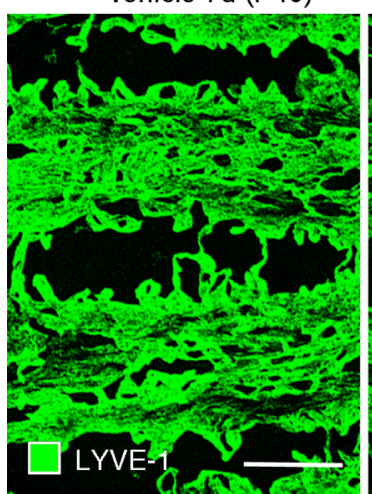

E LYVE-1 lymphatics

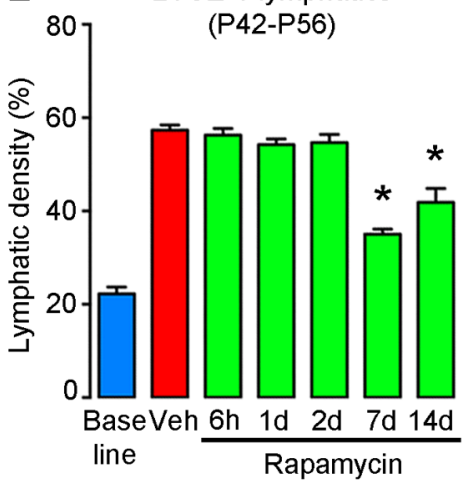

H

Numerical density of Prox1 cells in tracheal mucosa (P42-P56)

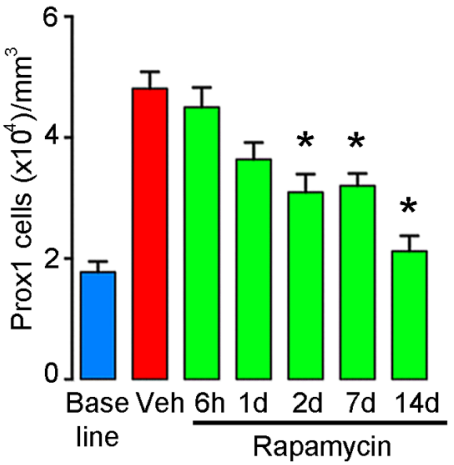

C Podocalyxin + LYVE-1 rapamycin $1 \mathrm{~d}(\mathrm{P} 43)$
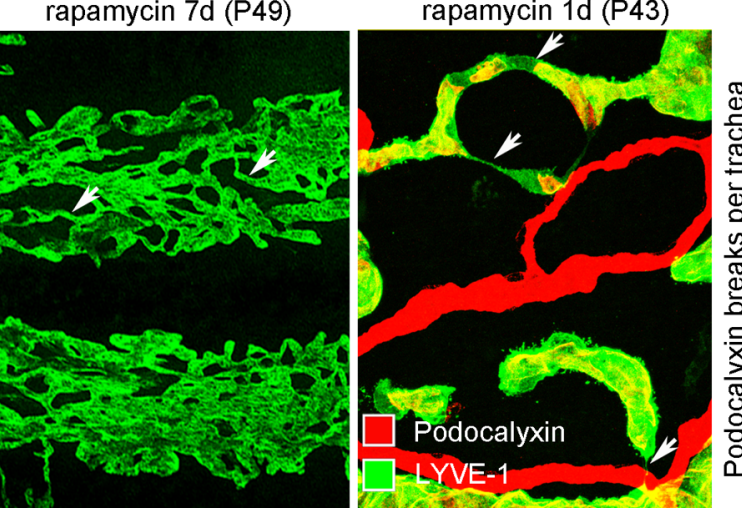

Podocalyxin breaks:

rapamycin 1d (P43)

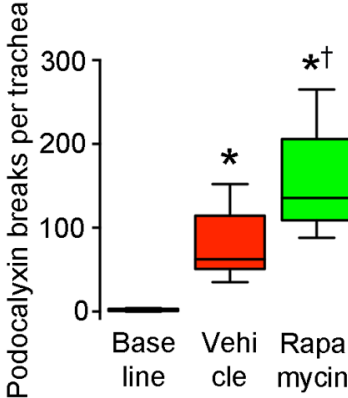

F Prox1 cells: vehicle 7d (P49)

G Prox1 cells: rapamycin 7d (P49)
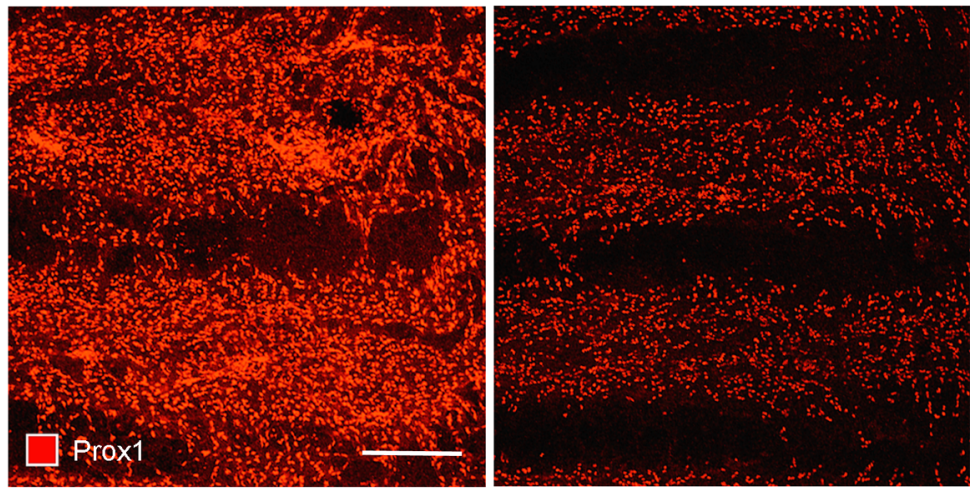

I Numerical density of Prox1 cells scaled to surface density of LYVE-1 lymphatics

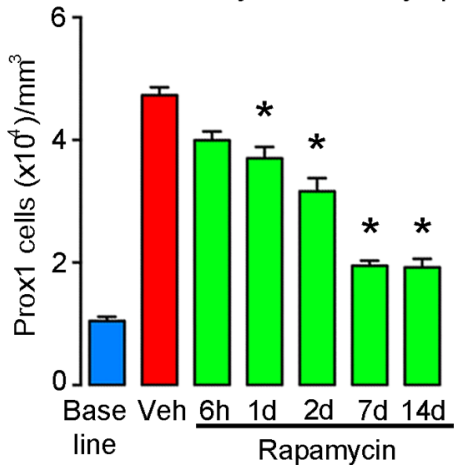

Figure 4. Time course and magnitude of rapamycin reversal of lymphangiectasia. (A and B) Lymphatic endothelial cells, stained for LYVE-1 after treatment for 7 days (P42-P49), form a sheet-like network after treatment with vehicle (A) but are more tubular after treatment with rapamycin (B, arrows). Scale bar: $200 \mu \mathrm{m}$. (C) Narrowing of lymphatics (arrows) after 1-day treatment with rapamycin. (D) Number of breaks in podocalyxin staining in lymphatics per trachea, reflecting lumen closure of lymphatics after rapamycin for 1 day (P42-P43) ( $n=6$ mice/group). ${ }^{*} P<0.05$ vs. baseline; ${ }^{\dagger} P<0.05$ vs. vehicle, ANOVA. Box and whisker plots show the median, first and third quartiles, and maximum and minimum. (E) Time course of lymphatic regression induced by rapamycin, as assessed by the extent of LYVE-1 staining in confocal microscopic projections of the tracheal surface. (F and $\mathbf{G})$ Lymphatic endothelial cells stained for Prox after treatment with vehicle or rapamycin for 7 days (P42-P49). (H) Time course of lymphatic regression induced by rapamycin, as assessed by the number of Prox1-positive nuclei in confocal projections of tracheal surface. (I) Prox1-positive nuclei per cubic millimeter of trachea, calculated as the product of $\mathbf{E}$ and $\mathbf{H}$. ${ }^{*} P<0.05$ vs. vehicle (red), ANOVA, $n=5$ mice/group (E-I).

Changes in mTOR signaling during lymphatic growth. mTOR signaling in lymphatics of CCSP/VEGF-C mice with or without doxycycline was assessed by immunofluorescence of three phosphorylated markers: phosphorylated mTOR (p-mTOR), phosphorylated S6 ribosomal protein (p-S6RP), and phosphorylated $4 \mathrm{E}$-binding protein 1 (p-4E-BP1) $(34,35)$. Staining for $\mathrm{p}-\mathrm{mTOR}$ was weak or absent in normal lymphatics (Figure 5A, no doxycycline) but was strong in most endothelial cells of growing lymphatics (Figure 5B, 3 days of doxycycline). Similarly, little or no p-S6RP staining was present in normal lymphatics (Figure 5C). 
After doxycycline (3 days), p-S6RP staining was strong in sprouts but was weak elsewhere in lymphatics (Figure 5D). Staining for p-mTOR and p-S6RP was also strong in tracheal epithelial cells.

Lymphatic endothelial cells stained for p-4E-BP1 were sparse in normal lymphatics (Figure 5E) but were clearly more abundant in CCSP/VEGF-C mice (Figure 5, F and G, 3 days of doxycycline). Most p-4E-BP1-positive cells were located at the tip of lymphatic sprouts, identified by costaining for LYVE-1 (Figure $5 \mathrm{H}$ ). Time-course studies revealed that, after doxycycline, p-4E-BP1-positive cells in lymphatics were most abundant at 2 days and then decreased to the baseline number at 7 days (Figure 5I). BrdU uptake experiments revealed that most p-4E-BP1-positive lymphatic endothelial cells (Prox1 labeled) were undergoing cell division (Figure $5 \mathrm{~J}$ ). In a prevention trial, rapamycin ( $20 \mathrm{mg} / \mathrm{kg}$ daily) completely blocked the increase in the number of p-4E-BP1-positive cells after doxycycline for 2 days (Figure $5, \mathrm{~K}$ and L). Few epithelial cells or other cell types had p-4E-BP1 staining.

$V E G F-C$ induction of lymphatic endothelial cell sprouting and proliferation. To build on evidence that rapamycin can block sprouting of lymphatic endothelial cells in vitro (20) and in zebrafish (36), we determined whether rapamycin can prevent lymphatic sprouting in CCSP/VEGF-C mice on doxycycline for 2 days (P21-P23). Lymphatic sprouts were abundant at 2 days but were reduced by $72 \%$ when doxycycline was given together with rapamycin (Figure 6, A and B). When doxycycline was given with rapamycin for 7 days (P21-P28), lymphangiectasia measured by LYVE-1 staining was reduced by $50 \%$ (Figure 6, C and D).

Proliferation of lymphatic endothelial cells in CCSP/VEGF-C mice on doxycycline was assessed by costaining of BrdU with Prox1 and/or LYVE-1. BrdU-labeled lymphatic endothelial cells were sparse or absent under baseline conditions (Figure 6E) but were abundant in CCSP/VEGF-C mice on doxycycline (Figure 6F). BrdU-labeled cells were most numerous after doxycycline for 1 day (15-fold of baseline, Figure $6 \mathrm{G}$ ) and were not restricted to sprouts (Figure 6F). BrdU labeling decreased after 3 days of doxycycline and was near baseline at 7 days (P28, Figure 6G). BrdU labeling was also found in some airway epithelial cells. In a prevention trial in CCSP/VEGF-C mice on doxycycline, rapamycin reduced BrdU labeling of lymphatics by $55 \%$ at 3 days and by $78 \%$ at 7 days (Figure $6 \mathrm{H}$ ) and reduced the number of Prox 1-positive cells by $87 \%$ at 3 days and by $52 \%$ at 7 days (Figure $6 \mathrm{I}$ ), indicating that the initial and almost complete growth prevention decreased during continued VEGF-C overexpression.

Rapamycin induction of lymphatic endothelial cell apoptosis. The effect of rapamycin on apoptosis of lymphatic endothelial cells was assessed by activated caspase-3 staining of tracheas from CCSP/VEGF-C mice on doxycycline for 3 days. Little or no staining was found in normal lymphatics (Figure 6J). In a prevention trial of doxycycline plus rapamycin for 3 days, scattered lymphatic endothelial cells (Prox1/LYVE-1 labeled) had activated caspase-3 staining (Figure $6 \mathrm{~K}$ ), but the increase in activated caspase- 3 staining after rapamycin was much smaller than the decrease in BrdU uptake.

VEGF-C induction of changes in Prox1 and VEGFR-3. Treatment with doxycycline (7 days) significantly increased Prox1 and VEGFR-3 immunofluorescence in endothelial cells of tracheal lymphatics in CCSP/VEGF-C mice but not in single-transgenic controls (Figure 7, A-E). Rapamycin (20 mg/kg, 2 days) did not decrease Prox1 or VEGFR-3 fluorescence in tracheal lymphatics of single-transgenic control mice (Figure 7, F-I).

\section{Mechanism of rapamycin reversal of established lymphangiectasia}

Lack of staining for mTOR signaling markers in lymphatic anomalies. Studies of mTOR signaling revealed that p-mTOR staining was weak or absent in lymphatics, both in vehicle-treated controls and after rapamycin (20 mg/kg), in the standard protocol (age P42-P56) (Supplemental Figure 4A), but was strong in epithelial cells (Supplemental Figure 4B). Staining for p-S6RP was also was weak or absent in lymphatics), with or without rapamycin (P42-P56) (Supplemental Figure 4C), and was strong in tracheal epithelial cells, smooth muscle, and some CD45-positive leukocytes (Supplemental Figure 4, D and E). Reduction of p-S6RP staining in CD45 leukocytes confirmed the efficacy of the dose of rapamycin (Supplemental Figure $4 \mathrm{~F})$. Staining for $\mathrm{p}-4 \mathrm{E}-\mathrm{BP} 1$ was weak or absent in lymphatics under these conditions regardless of treatment (P42-P56) (Supplemental Figure 4G). The contribution of technical factors to the lack of staining of mTOR signaling markers in lymphatics was excluded by positive staining of epithelial cells and inhibition of that staining by blocking peptides (Supplemental Figure $4 \mathrm{H}$ ).

Lack of endothelial cell proliferation in lymphatic anomalies. As in the prevention trials, we assessed the effect of rapamycin on lymphatic endothelial cell proliferation in reversal trials. BrdU labeling of Prox1/LYVE-1positive lymphatic endothelial cells was sparse or absent before initiation of rapamycin (P42) (Supplemental 
Markers of mTOR signaling in lymphatics at baseline, after doxycycline, and in prevention trial
A p-mTOR cells $\mathbf{B}$
C $\mathrm{p}$-S6RP cells
p-S6RP cells

B $\begin{gathered}\mathrm{p} \text {-mTOR cells } \\ \text { doxycycline } 3 \mathrm{~d}(\mathrm{P} 24)\end{gathered}$

Control
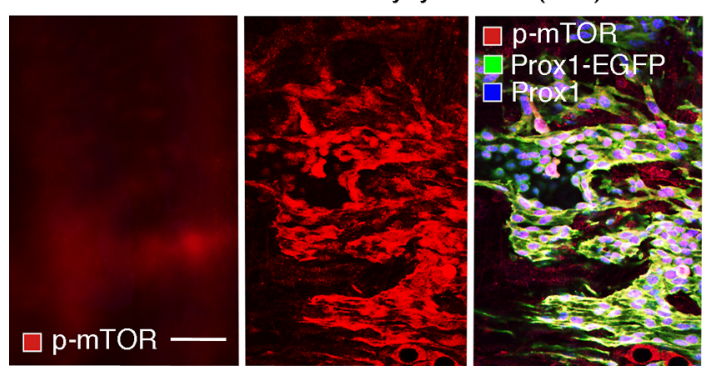

Control

doxycycline 3d (P24)

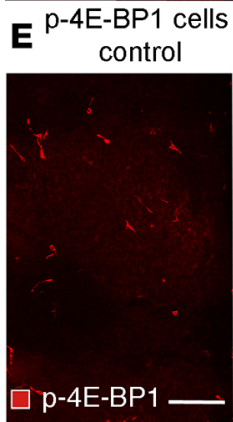

$\mathbf{F}$
p-4E-BP1 cells

doxycycline 3d (P24)
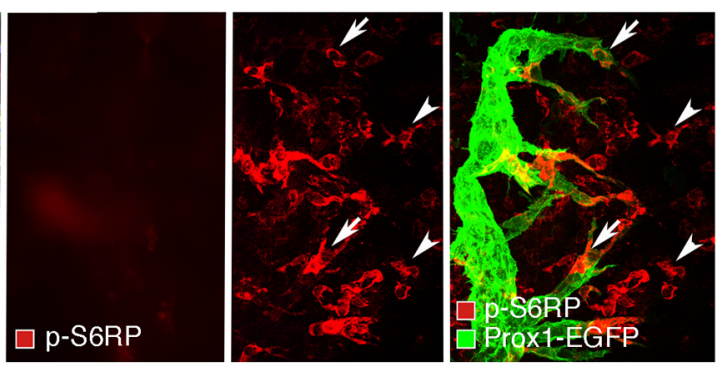

G

p-4E-BP1 cells 3d doxycycline (P24)
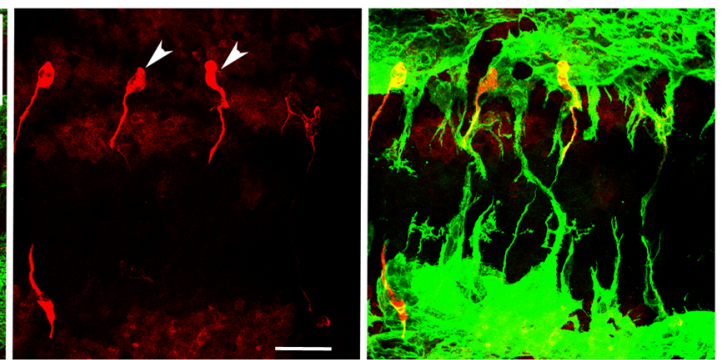

$\mathbf{J}$

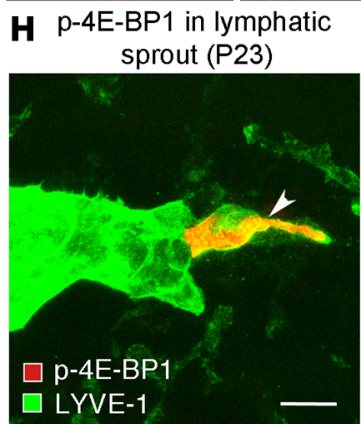

$\mathbf{K}$

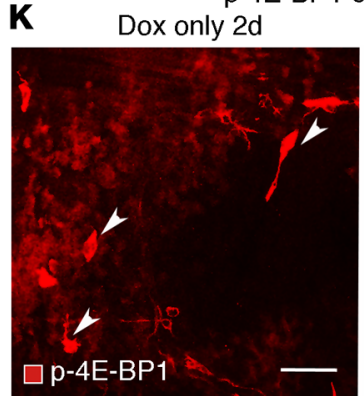

p-4E-BP1 cells change during doxycycline
BrdU-labeled p-4E-BP1/Prox1 cells doxycycline 3 days (P24)

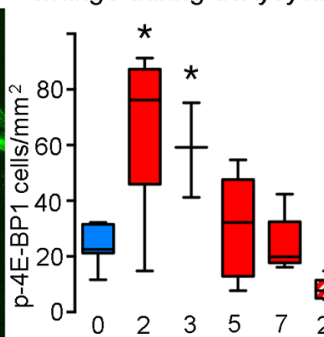

$\begin{array}{llllll}0 & 2 & 3 & 5 & 7 & 21\end{array}$ p-4E-BP1 cells (P21-P23)

Dox + rapamycin 2d

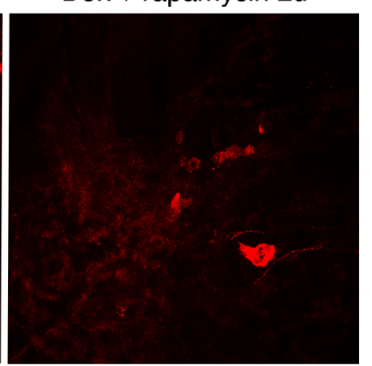

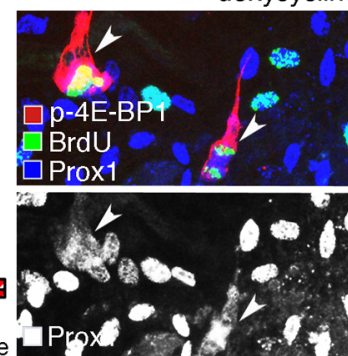

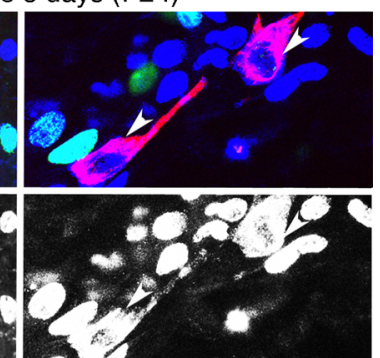

p-4E-BP1-positive cells

$\mathbf{i n}$ in lymphatics

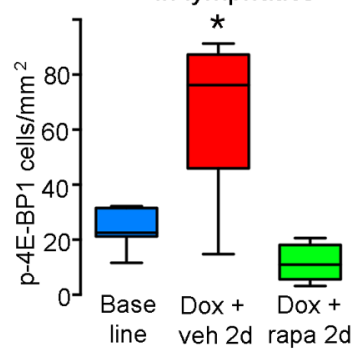

Figure 5. Staining for p-mTOR, p-S6RP, and p-4E-BP1 in growing lymphatics. (A) Absence of p-mTOR staining in lymphatics of normal trachea. (B) Uniformly strong p-mTOR staining in lymphatics. The epithelium was excluded from the confocal projection of the tracheal whole mount. (C) Absence of $p$-S6RP staining in lymphatics of normal trachea. (D) Strong p-S6RP staining in lymphatic sprouts (arrows) in CCSP/VEGF-C mice after doxycycline (3 days). Arrowheads mark p-S6RP staining of other cell types. (E) Absence of p-4E-BP1 staining in lymphatics of normal trachea. (F) Strong p-4E-BP1 staining in a subset of lymphatic endothelial cells in CCSP/VECF-C mice on doxycycline ( 3 days). (C) The boxed region in $\mathbf{F}$ shown at higher magnification. Arrowheads mark p-4E-BP1-positive cells. (H) Phospho-4E-BP1 staining in a lymphatic sprout (arrowhead) in CCSP/VEGF-C mice on doxycycline (2 days, P23). (I) Transient increase in number of p-4E-BP1-stained cells during doxycycline exposure (red boxes, P21-P28) and 14 days after withdrawal of doxy-

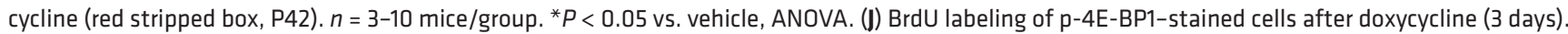
BrdU labeling (green) in lymphatic endothelial cells is more abundant than p-4E-BP1 (red). Prox1 (white in bottom panels) is not restricted to the nuclei of dividing cells (arrowheads, left panels). Some p-4E-BP1-stained cells lack BrdU labeling (arrowheads, right panels). (K) Numerous p-4E-BP1-stained cells were observed after doxycycline alone (2 days, P21-P23) but not when doxycycline was given concurrently with rapamycin. (L) An increased number of p-4E-BP1-stained cells (red box) was observed after treatment with doxycycline (2 days, P21-P23). The increase was prevented when doxycycline was given concurrently with rapamycin ( $20 \mathrm{mg} / \mathrm{kg}$, green box). ${ }^{*} P<0.05$ vs. baseline, ANOVA, $n=5$ mice/group. Scale bar: $50 \mu \mathrm{m}(\mathbf{A}-\mathbf{D}, \mathbf{G}$, and $\mathbf{K}) ; 200 \mu \mathrm{m}(\mathbf{E}$ and $\mathbf{F}) ; 10 \mu \mathrm{m}(\mathbf{H})$. Box and whisker plots show the median, first and third quartiles, and maximum and minimum. 
A
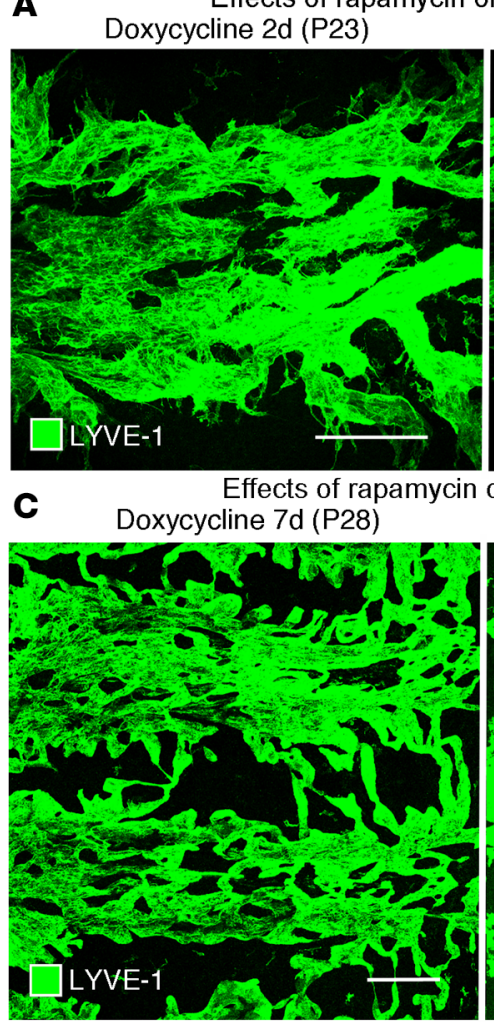

E Absent BrdU in lymphatics

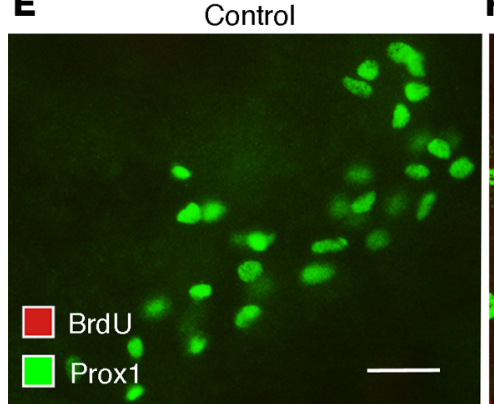

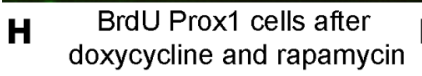

Doxycycline + rapamycin 2d (P23)

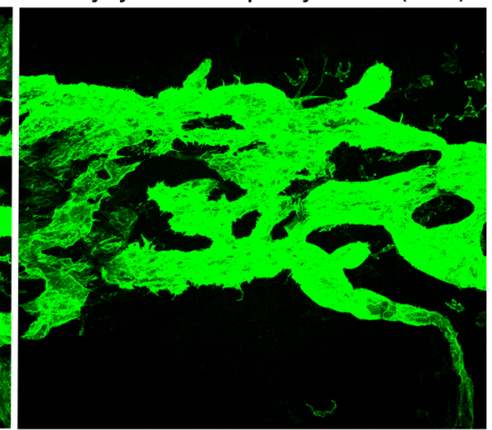

Doxycycline + rapamycin 7d (P28)

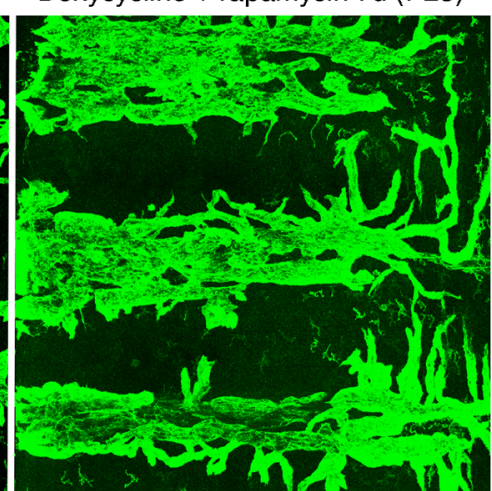

BrdU in lymphatics

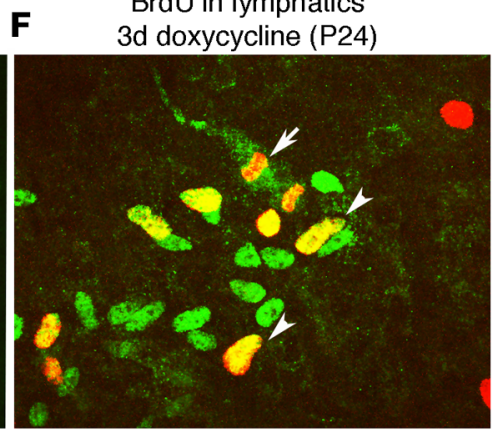

Prox 1 cells after

xycycline and rapamycin

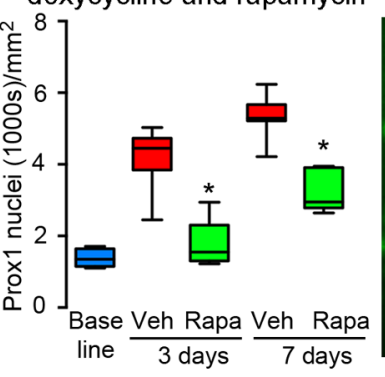

B Lymphatic sprouts (P23)

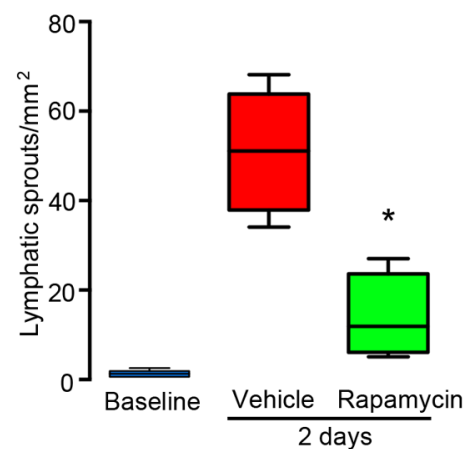

LYVE-1 lymphatics (P28)

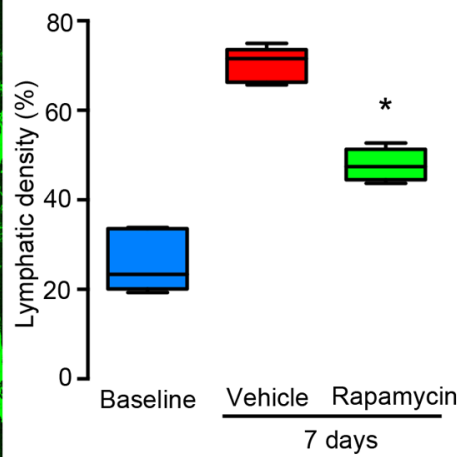

BrdU Prox1 cells during

G doxycycline (P21-P28)

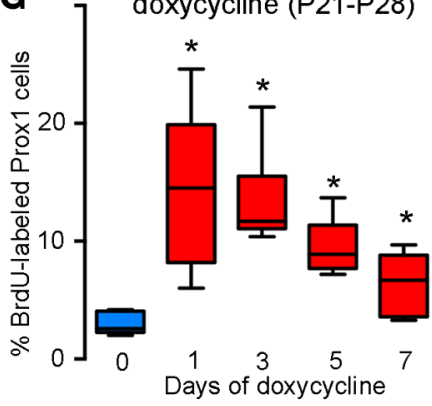

K Activated caspase-3

after rapamycin (P24)

Figure 6. Effect of rapamycin on lymphatic sprouting and proliferation in tracheas of CCSP/VEGF-C mice. (A) Lymphatic sprouts after doxycycline (left) but not after doxycycline plus rapamycin (right) (2d, P21-P23). (B) Measurements of lymphatic sprouts. Baseline, single-transgenic mice (P21). ${ }^{*} P<0.05$ vs. vehicle, ANOVA. (C) Lymphangiogenesis after doxycycline (left) but not after doxycycline plus rapamycin (right) (7d, P21-P28). (D) Measurements of lymphatic density. ${ }^{*} P<0.05$ vs. vehicle, ANOVA. (E) No BrdU uptake by lymphatics in control mouse, compared with (F) BrdU-labeled lymphatics (arrowheads) after doxycycline (3d). Dividing lymphatic endothelial cell (arrow). (C) BrdU labeling of lymphatics after doxycycline for 1-7 days (P21-P28). ${ }^{*} P<0.05$ vs. baseline, ANOVA. (H) Rapamycin suppression of BrdU labeling after doxycycline for 3 or 7 days (P24 or P28), expressed as a percentage of Prox1-positive cells. (I) Reduction in Prox1-positive cells after doxycycline plus rapamycin for 3 days (87\% reduction, P24) or 7 days (52\% reduction, P28). (J) No activated caspase-3 staining in control lymphatics, compared with (K) staining of fragmented Prox1-positive cells (arrows) after doxycycline plus rapamycin (3d, P21 to P24). $n=5$ mice/group. Scale bar: $100 \mu \mathrm{m}$ (A); $200 \mu \mathrm{m}$ (C); $20 \mu \mathrm{m}$ (E, F, J, and K). Box and whisker plots show the median, first and third quartiles, and maximum and minimum. 
Prox1 and VEGFR-3 intensity in lymphatics of control and CCSP/VEGF-C mice after doxycycline (7d)

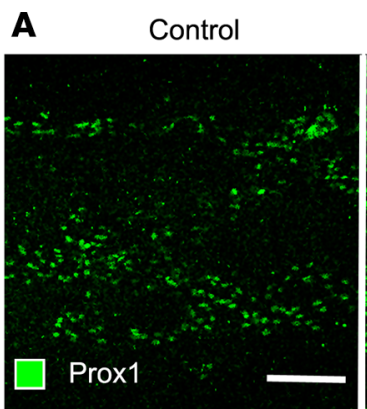

CCSP/NEGF-C (P28)

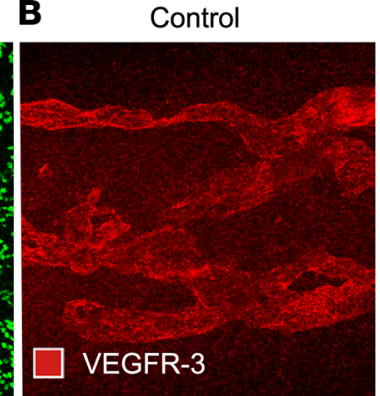

CCSP/VEGF-C (P28)

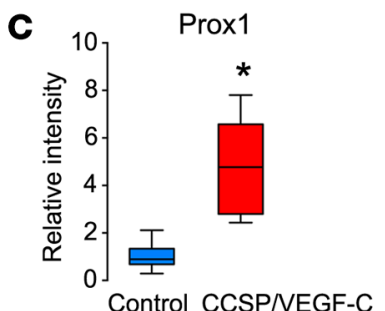

D

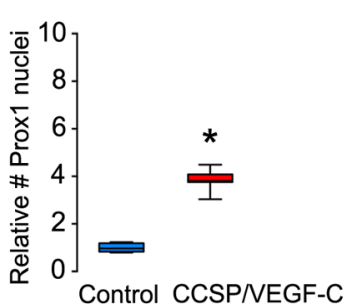

E
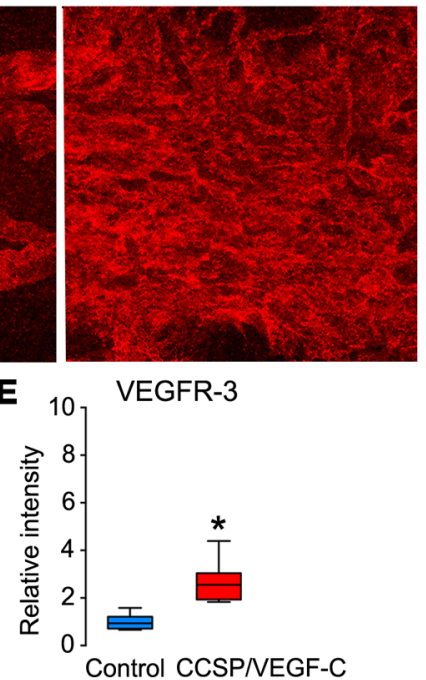

Prox 1 and VEGFR-3 intensity in lymphatics of control mice after rapamycin (2d)
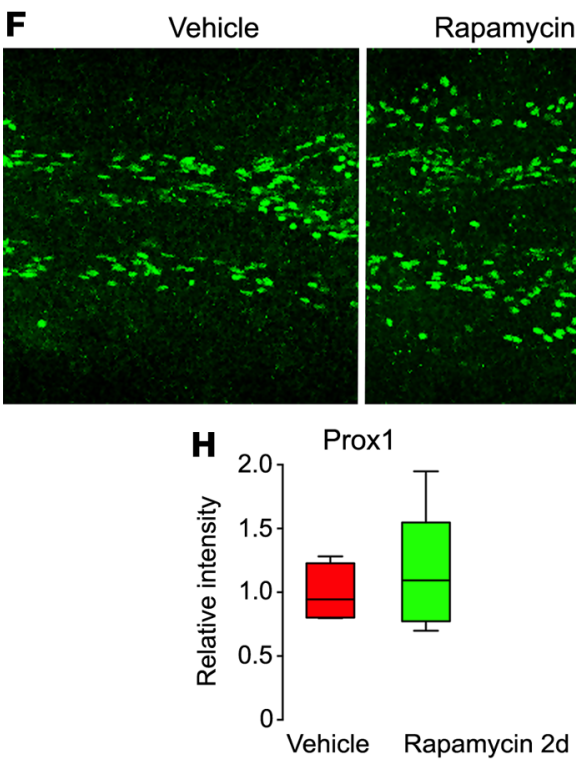

Rapamycin 2d (P44)
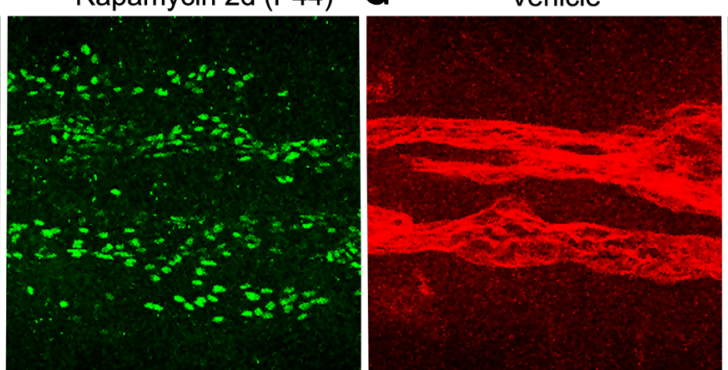

Rapamycin 2d (P44)

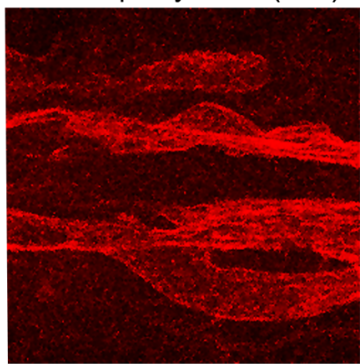

VEGFR-3

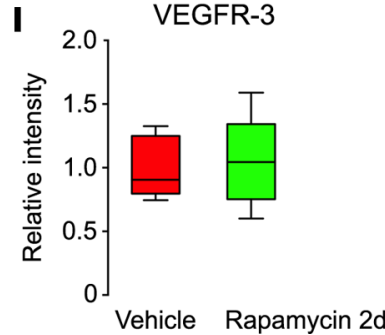

Figure 7. Effects of doxycycline and rapamycin on Prox1 and VEGFR-3 in lymphatics of normal mice. (A and B) Confocal micrographs comparing Prox1 (A) and VEGFR-3 (B) staining of lymphatics in the trachea of control mouse (left, single-transgenic CCSP) and a CCSP/VEGF-C mouse (right) after doxycycline (7 days, P21-28). Images were photographed with matching brightness and contrast settings. (C-E) Intensity of Prox 1 fluorescence (C), number of Prox1-positive nuclei (D), and intensity of VEGFR-3 fluorescence (E) under same conditions as in $\mathbf{A}$ and $\mathbf{B}$. ${ }^{*} P<0.05$ vs. single-transgenic control, ANOVA; $n=5-6$ mice/group. (F-I) Confocal micrographs comparing Prox1 (F) and VEGFR-3 (G) fluorescence in lymphatics and corresponding measurements ( $\mathbf{H}$ and $\mathbf{I}$ ) in normal mice after treatment with vehicle or rapamycin (2 days, P21-P23). Scale bar: $100 \mu \mathrm{m}$. Box and whisker plots show the median, first and third quartiles, and maximum and minimum.

Figure 5A) and was similarly sparse after rapamycin for 14 days (P56) (Supplemental Figure 5, B and C). Scattered BrdU-positive cells of other types (no Prox1/LYVE-1 staining) served as a positive control for the method (Supplemental Figure 5, B and C).

Lack of endothelial cell apoptosis in lymphatic anomalies. Activated caspase-3 staining of Prox1/LYVE1-positive cells was assessed to determine the contribution of apoptosis to rapamycin-induced reversal of lymphangiectasia. Little or no staining for activated caspase-3 was found in lymphatic endothelial cells after vehicle for 14 days or rapamycin for 1 or 14 days (P43 or P56) (Supplemental Figure 5, D-G). As a positive control, scattered epithelial cells, smooth muscle cells, and leukocytes had activated caspase-3 staining (Supplemental Figure 5, G and H). 
Effect of rapamcyin on fluorescence intensity of Prox1, VEGFR-3, and LYVE-1 in lymphatics in CCSPNEGF-C mice

A
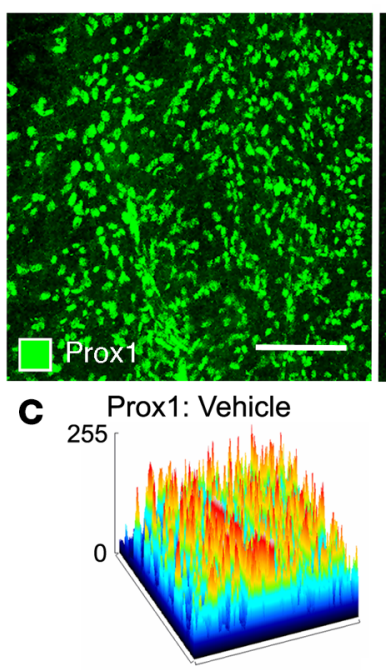

Rapamycin 2d (P44)

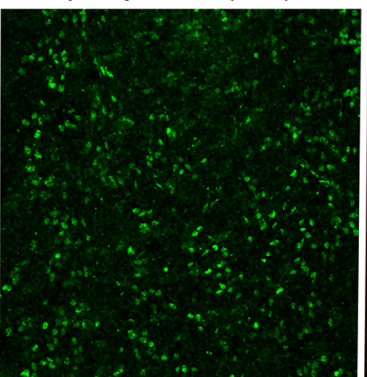

Prox1: Rapamycin 2d 255
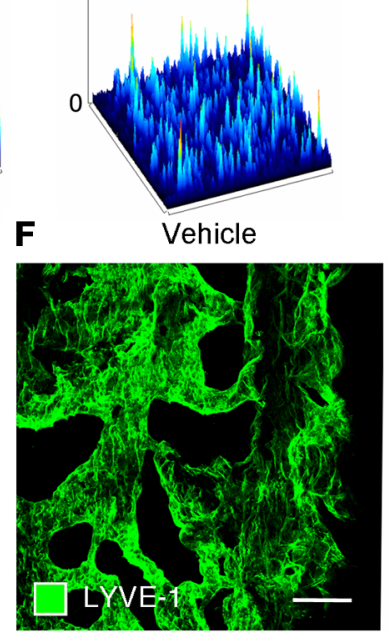

G

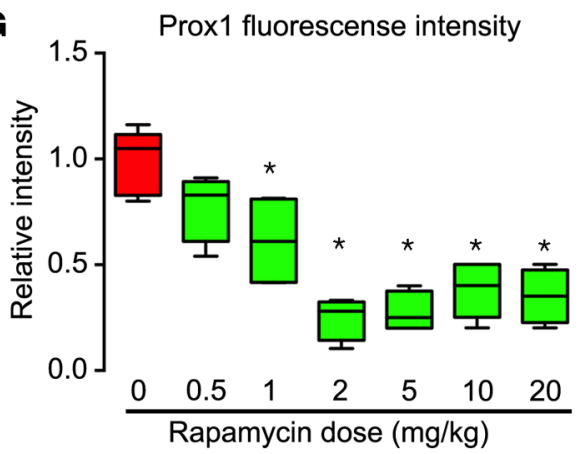

B
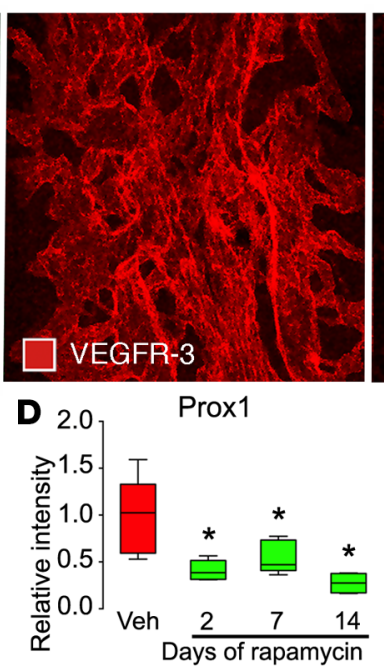

Rapamycin 14d (P56)

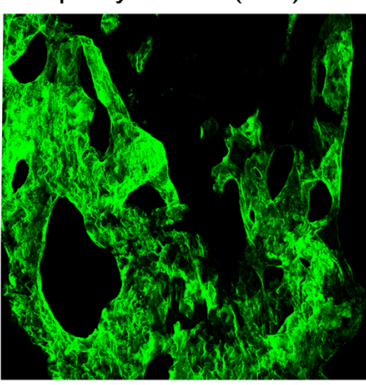

Rapamycin 2d (P44)

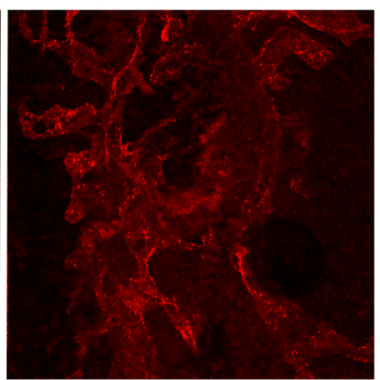

E 2.0 VEGFR-3

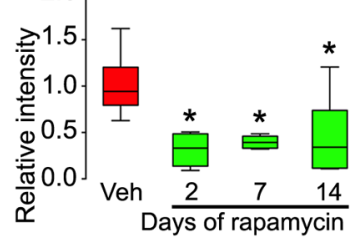

H

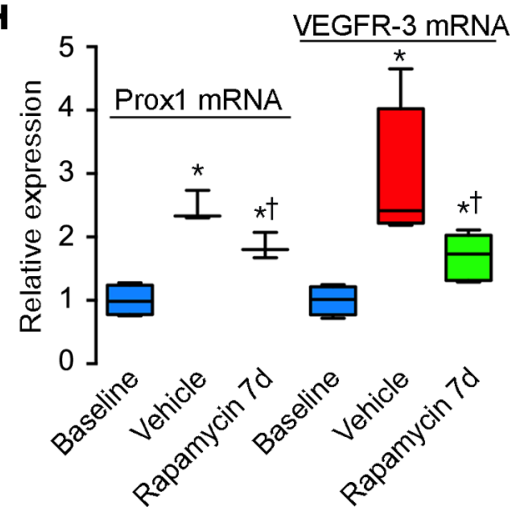

Figure 8. Effects of rapamycin on Prox1, VEGFR-3, and LYVE-1 in lymphatics of CCSP/VEGF-C mice. (A and B) Confocal micrographs comparing Prox1 (A) and VEGFR-3 (B) immunofluorescence in tracheas of CCSP/VEGF-C mice treated with vehicle or rapamycin (2 days, P42-P44). Images photographed with matching brightness and contrast settings. (C) Surface contour plots of intensity of Prox1 fluorescence in $\mathbf{A}$ after treatment with vehicle or rapamycin. Prox1 (D) and VEGFR-3 (E) fluorescence intensities in CCSP/VEGF-C mice treated, as in $\mathbf{A}$ and $\mathbf{B}$. (F) Confocal micrographs of LYVE-1 immunofluorescence in tracheas of CCSP/VEGF-C mice, showing no difference in intensity after treatment with vehicle or rapamycin (14 days, P42-P56). (C) Rapamycin dose-response effect on Prox1 fluorescence intensity. ${ }^{*} P<0.05$ vs. vehicle, ANOVA, $n=4-6$ mice/group. (H) qRT-PCR measurements of Prox1 and VEGFR-3 expression in tracheas of CCSP/VEGF-C mice on vehicle or rapamycin (7 days, P42-P49). ${ }^{*} P<0.05$ vs. baseline, ${ }^{\dagger} P<0.05$ vs. vehicle, ANOVA, $n=3-6$ mice/ group. Scale bars: $200 \mu \mathrm{m}$ (A and B); $50 \mu \mathrm{m}$ (F). Box and whisker plots show the median, first and third quartiles, and maximum and minimum.

Rapamycin suppression of Prox1 and VEGFR-3. The lymphatic endothelial cell differentiation markers Prox1 and VEGFR-3 were suppressed by treatment of CCSP/VEGF-C mice with rapamycin (20 mg/ $\mathrm{kg}, 2$ days, P42-P44) (Figure 8, A-C). Similar reductions were found after rapamycin for 2, 7, or 14 days (P42-P56) (Figure 8, D and E). Mean Prox 1 fluorescence was reduced by 59\% at 2 days, $45 \%$ at 7 days, and $73 \%$ at 14 days (Figure 8D and Supplemental Figure 3G), and mean VEGFR-3 fluorescence was reduced by $68 \%$ at 2 days, $61 \%$ at 7 days, and $56 \%$ at 14 days (Figure $8 \mathrm{E}$ and Supplemental Figure $3 \mathrm{H}$ ). LYVE-1 
fluorescence was not reduced at 14 days (Figure 8F). Rapamycin dose-response studies revealed that Prox1 fluorescence was reduced at doses of $1 \mathrm{mg} / \mathrm{kg}$ or greater (Figure $8 \mathrm{G}$ ).

Doxycycline increased the expression of Prox1 mRNA by 2.5 -fold and VEGFR-3 mRNA by 3-fold in CCSP/VEGF-C mice (doxycycline $0.01 \mathrm{mg} / \mathrm{ml}$, P21-P28; washout P28-P42; treatment with vehicle P42P49) compared with normal controls (Figure 8H). Treatment of CCSP/VEGF-C mice with rapamycin (20 $\mathrm{mg} / \mathrm{kg}, 7$ days, P42-P49) reduced tracheal expression of Prox 1 mRNA by $42 \%$ and VEGFR-3 mRNA by $66 \%$ (Figure $8 \mathrm{H})$.

\section{Discussion}

To obtain pathophysiologic insights into the action of rapamycin on lymphatic anomalies, we used CCSP/ VEGF-C-transgenic mice that overexpress VEGF-C in airway epithelial cells and develop lymphatic abnormalities similar to congenital pulmonary lymphangiectasia. The model was used to test the reversibility of established lymphangiectasia by VEGFR-2- and VEGFR-3-blocking antibodies, Notch signaling inhibitor DAPT, $\beta$ receptor inhibitor propranolol, autophagy inhibitor chloroquine, antiinflammatory steroid dexamethasone, and mTOR inhibitor rapamycin. Of these, rapamycin was the only effective agent, and its efficacy was not increased by the other agents. Dose-response studies revealed that the highest dose (20 mg/kg i.p. daily) was required to achieve maximal reversal (76\%) of lymphangiectasia at a stage when there was little or no endothelial cell proliferation. Structural changes in lymphatics were evident after 1 day of rapamycin, but no caspase-dependent apoptosis was found. Rapamycin did, however, rapidly reduce expression of Prox1 and VEGFR-3 mRNA and protein. The findings confirmed the efficacy of rapamycin in treatment of lymphatic anomalies and revealed that the action was rapid, possibly involving suppression of Prox1 transcriptional activity. Rapamycin had no apparent effects on normal lymphatics.

Attributes and limitations of the CCSP/VEGF-C mouse model of lymphangiectasia. In the present work, we optimized the start age, dose, and duration of doxycycline treatment to induce VEGF-C overexpression and the washout period following it to create conditions in which the effect of therapeutics on established lymphangiectasia could be tested. Among the attributes of this approach, the model made it possible to (a) induce lymphangiectasia in mice that were otherwise normal and had genetically normal lymphatics; (b) promote lymphatic growth with the natural lymphangiogenic factor VEGF-C; (c) separate effects on lymphatic growth from those on regression by distinguishing changes in endothelial cell proliferation from changes in cell survival; and (d) determine the dose response and time course of action of rapamycin under these conditions.

By assessing lymphatics in situ, instead of dissociating the tissue for flow cytometry, we were able to examine the abnormalities in their natural setting (6). LYVE-1 staining revealed the overall extent of lymphatics, and Prox1 staining revealed the number of lymphatic endothelial cells in layers of overlapping lymphatics. Macrophages that expressed LYVE-1 did not express Prox1, and neuroendocrine cells that expressed Prox1 did not express LYVE-1.

Other models involving gene deletions or implantation of cells from human lymphatic malformations have been used to mimic human lymphatic disorders (37-40). These models have proven useful in characterizing these conditions but are yet to be used to study the regression of abnormal lymphatics.

Among the limitations of the CCSP/VEGF-C model, lymphatic growth is driven by VEGF-C from airway epithelial cells, which is an unlikely mechanism of lymphatic anomalies in humans. VEGF-C is expressed in some human lymphatic anomalies $(41,42)$, and VEGFR-3 and neuropilin-2 are overexpressed in endothelial cells of vascular malformations with lymphatic features (43). However, VEGF-C from epithelial cells may not mimic all consequences of mutations in AKT, PI3K, RAS, or other genetic defects associated with lymphatic malformations in humans (2). In addition, in our mouse model, the therapeutic agents were tested on established lymphangiectasia, when lymphatic endothelial cells were no longer proliferating. In contrast, in some human lymphatic malformations the endothelial cells continue to grow. In the latter case, rapamycin should be even more efficacious by blocking proliferation as well as causing regression. As lymphatic anomalies in CCSP/VEGF-C mice are restricted to the respiratory tract, the model does not mimic conditions in which multiple organs are involved.

Resistance of lymphatics to regression. Historical studies revealed that skin lymphatics induced to grow by chemical irritants can persist for many months after exposure (44). Subsequent experiments confirmed that lymphatic growth resists regression after (a) inflammation resolves (13), (b) growth factor overexpression is switched off $(6,45)$, and (c) genetic deletion of VEGFR-2 and VEGFR-3 $(46,47)$. 
However, rapid spontaneous regression of lymphatics has been reported in corneal inflammation (48), mammary gland regression after lactation (49), lymph node involution (50), adipose tissue after stopping VEGF-D overexpression (51), and intestinal lacteals after genetic deletion of VEGF-C (46). Further studies are needed to learn the mechanisms underlying the differences in dependence of lymphatics on environment survival factors.

Rationale for therapeutic approaches tested for regression of lymphangiectasia. Our rationale for comparing the efficacy of six therapeutic strategies for reversing established lymphangiectasia was based on published reports of their use in prevention or treatment of lymphatic malformations, hemangiomas, and related conditions (11-30). Inhibition of VEGFR-2 and VEGFR-3 signaling by function-blocking antibodies prevents lymphatic growth in inflammation and in CCSP/VEGF-C mice $(6,13)$. Some spontaneous lymphatic regression occurred during the washout period in CCSP/VEGF-C mice, when VEGF-C expression decreased, but little regression occurred thereafter. VEGFR-3 signaling blockade results in regression of lymphatics in the heart, intestine, and skin of young, but not older, mice (52).

Propranolol has been used in the treatment of hemangioma (53), lymphangiectasia (16), lymphangiomatosis (24), and other lymphatic abnormalities (2) but had little or no effect on lymphangiectasia in CCSP/VEGF-C mice. Responses in some pediatric patients with lymphatic malformations indicate that propranolol is effective under some conditions (54). Lesions with endothelial cell proliferation driven by VEGF-A, instead of VEGF-C, could be more responsive (55).

Suppression of Notch signaling has both prolymphangiogenic and antilymphangiogenic effects. Inhibition of Notch signaling impairs lymphatic development in zebrafish (56) but results in overgrowth of lymphatics in developing mice $(57,58)$ and promotes VEGF-driven lymphatic sprouting in adult mice (59). Inhibition of Notch signaling reduces lymphangiogenesis associated with wound healing (60) but did not reverse lymphangiectasia in our experiments.

Because activation of autophagy promotes cell survival $(18,26)$, we reasoned that inhibition of autophagy could impair survival of abnormal lymphatic endothelial cells. However, chloroquine did not reverse lymphangiectasia in our model. Consistent with the antiinflammatory effects of glucocorticoids, dexamethasone prevents lymphangiogenesis in multiple settings $(13,19)$. Yet dexamethasone does not reverse lymphatic growth in inflammation (13) and did not reverse lymphangiectasia in our model.

Rapamycin was the only agent of the six tested that reversed established lymphangiectasia. Rapamycin also prevented VEGF-C-driven lymphatic endothelial cell sprouting and proliferation in CCSP/VEGF-C mice. Sustained high blood levels after the $20 \mathrm{mg} / \mathrm{kg}$ daily dose required to reduce lymphangiectasia could have contributed to efficacy. It also raised the question of possible toxicity. Although dose-dependent body weight loss occurred, as reported by others (31), survival was $100 \%$ with all doses. Doses as high as 400 $\mathrm{mg} / \mathrm{kg}$ are reported to be well tolerated in mice $(29,30)$. Dose-related effects of rapamycin on the thymus and blood lymphocytes were observed, as expected, but hematocrit, white blood cell count, and platelet count were little changed $(32,33)$. Efficacious rapamycin levels in mouse blood were higher than typical therapeutic concentrations of $5-15 \mathrm{ng} / \mathrm{ml}$ in human blood (27), but clearance was much faster, with a blood half-life of 6.5 hours in mice compared with about 60 hours in humans (33).

Rapamycin prevention of lymphatic growth and reversal of lymphatic anomalies. The efficacy of rapamycin in reversing lymphangiectasia in CCSP/VEGF-C mice fits with compelling data from patients. mTOR signaling is upregulated in patients with lymphangioleiomyomatosis (LAM) with tuberous sclerosis complex gene mutations $(2,61)$, and rapamycin is efficacious in the treatment of $\operatorname{LAM}(18,27,61,62)$. In preclinical models, rapamycin prevents or inhibits lymphangiogenesis in zebrafish (36) and in mouse skin flaps, kidney allograft injury, and tumor metastasis $(63,64)$. Rapamycin also suppresses growth of lymphatic endothelial cells in vitro (65).

VEGFR-2 and VEGFR-3, the receptors for VEGF-C and VEGF-D, are upstream of PI3K/AKT/ mTOR $(2,66,67)$. Newborn mice with PI3K mutations have lymphatic abnormalities and chylous ascites (68). AKT signaling is required for lymphatic network formation and remodeling (69). AKT is hyperphosphorylated in lymphatic endothelial cells from human lymphatic anomalies with PI3K mutations (20). Lymphatic growth can be prevented in multiple settings by targeting PI3K (70). Inhibition of PI3K/AKT signaling could augment the efficacy of rapamycin.

The finding that rapamycin did not completely convert the malformed lymphatics in our lymphangiectasia model into normal lymphatics implicates other signaling pathways in the lymphatic endothelial cell phenotype and survival. Although AKT/mTOR is regarded as the dominant pathway for VEGF-C-mediated 
lymphatic growth, ERK/MAPK, JNK, and other pathways can contribute (38, 71, 72). Moreover, VEGF-C blockade may not fully suppress VEGFR-3 signaling because of crosstalk between receptors (71). Although inhibition of multiple signaling pathways may be necessary to obtain complete and sustained lymphatic regression, none of the agents tested augmented the regression of lymphangiectasia by rapamycin.

Cellular mechanisms of rapamycin on lymphatic growth and regression. As possible mechanisms of rapamycin-induced reversal of lymphangiectasia in CCSP/VEGF-C mice, inhibition of proliferation or induction of caspase-dependent apoptosis had little contribution because of the lack of BrdU uptake and activated caspase-3 staining in lymphatics at the onset (P42) or end (P56) of rapamycin treatment. This raises the possibility that rapamycin induces caspase-independent cell death in established lymphangiectasia, as found in other settings $(73,74)$. In prevention studies, rapamycin readily reduced VEGF-C-induced sprouting and proliferation of lymphatic endothelial cells and increased apoptosis in CCSP/VEGF-C mice on doxycycline, consistent with evidence from in vitro studies $(65,75)$. Although rapamycin reduced proliferation and increased apoptosis of lymphatic endothelial cells in tracheas of CCSP/VEGF-C mice with high VEGF-C expression during doxycycline exposure (P21-P28), it did not have these effects when started 14 days after cessation of doxycycline (P42). In regression studies, rapamycin normalized some regions of the abnormal lymphatics and narrowed the diameter and closed the lumen of others but did not induce caspase-dependent apoptosis.

Rapamycin downregulation of Prox 1 and VEGFR-3 in abnormal lymphatics. Rapamycin reduced Prox1 and VEGFR-3 mRNA expression and protein levels in abnormal lymphatics in CCSP/VEGF-C mice but had no noticeable effect on either one in normal lymphatics. The findings reflect changes in the amount of Prox1 and VEGFR-3 per cell and in the total number of lymphatic endothelial cells. Rapamycin downregulates expression of VEGFR-3 in lymphatic endothelial cells in vitro (65) and Prox1 in mouse liver (76).

Prox1 is a key regulator of lymphatic endothelial cell differentiation, maintenance, and survival (77). During lymphatic development, Prox1 and VEGFR-3 participate in a positive feedback loop, such that downregulation of one reduces the other (78). This loop is important for maintenance of the identity and number lymphatic endothelial cell progenitors (78). Downregulation of Prox1 and VEGFR-3 could render lymphatic endothelial cells more susceptible to rapamycin-induced regression, but the dose-response studies revealed that the threshold for changes in Prox1 is lower than that for lymphatic regression. Although genetic ablation of VEGFR-3 in lymphatics of adult mice is followed by fewer Prox1-positive lymphatics 3 months later (47), the abundance of lymphatics in lymph nodes, ears, or tracheas is not reduced 6 months after global deletion of the Vegfc gene in adult mice (46). Our finding of no reduction in established lymphangiectasia after inhibition of VEGFR-2 and VEGFR-3 signaling for 2 weeks is consistent with the latter report.

Significance of mTOR activity in growing lymphatics. p-mTOR and p-S6RP were widely expressed in growing lymphatics. p-4E-BP1 was also found in growing lymphatics but was limited to dividing lymphatic endothelial cells. Rapamycin strongly inhibited p-S6RP and p-4E-BP1 staining as well as BrdU uptake in growing lymphatics, consistent with cell cycle inhibition in $\mathrm{G}_{1}(34,35)$. The contrasting distributions of p-mTOR, p-S6RP, and p-4E-BP1 in growing lymphatics is consistent with the differential regulation of these components of mTOR signaling (79).

Lymphatic endothelial cells in established lymphangiectasia (P42-P56) lacked the three markers of mTOR activity, regardless of treatment. Responsiveness to rapamycin in the absence of detectable staining for p-mTOR, p-S6RP, or p-4E-BP1 indicates that the level of mTOR signaling in abnormal lymphatics that regress was below the threshold of detection by the approach used.

Translational implications. Rapamycin and related mTORC1 inhibitors are being used with promising results in the treatment of lymphatic malformations $(4,80-82)$, which build on what is known from the treatment of patients with LAM $(27,83)$. Favorable responses provide incentive for learning more about the actions of mTORC1 inhibitors in the treatment of lymphatic anomalies. Devising efficacious management of these conditions has been challenging because of their varied nature, poorly understood pathophysiology, and rarity (1-3).

Pulmonary lymphangiectasia in CCSP/VEGF-C mice offers an opportunity to elucidate some previously unrecognized features. Distinctively, rapamycin was the only agent of the six tested that resulted in significant regression of lymphangiectasia. Rapamycin had rapid and robust actions that led to $76 \%$ regression over 7 days. The regression was associated with striking reduction of Prox 1 and VEGFR-3 in the abnormal lymphatic endothelial cells. These changes were, however, not accompanied by induction of 
caspase-dependent apoptosis of these cells. Lymphatic endothelial cell proliferation was not suppressed because it was already negligible at the onset of treatment. Because rapamycin did reduce cell proliferation during VEGF-C-driven lymphatic growth, the agent should be even more beneficial for lymphatic malformations that are enlarging.

Conclusions. From studies of pulmonary lymphangiectasia in CCSP/VEGF-C mice, we conclude that rapamycin not only prevents the growth of abnormal lymphatics, but also induces partial regression of established lymphangiectasia, without apparent effects on normal lymphatics. The regression is accompanied by reduction in Prox1 and VEGFR-3 but not by caspase-dependent apoptosis of lymphatic endothelial cells. Rapamycin does not suppress endothelial cell proliferation in established lymphangiectasia where the level of proliferation is already low. The effects of rapamycin in this mouse model fit with clinical observations in patients with lymphatic anomalies and provide opportunities to identify additional features of the pathophysiology and management of these challenging conditions.

\section{Methods}

Mice

Double-transgenic CCSP-rtTA/tetO-VEGF-C (CCSP/VEGF-C) mice of both sexes in a FVB/N background were generated by breeding Clara cell secretory protein reverse tetracycline transactivator (CCSP-rtTA) driver mice (The Jackson Laboratory, strain 006222, line 1) with tetracycline operator-mVEGF-C (tetO-mVEGF-C) responder mice. Doxycycline induces rapid, reversible, and dose-dependent transgenic expression of fulllength mouse VEGF-C in Clara/Club cells of the airway epithelium and alveolar type II cells as described previously (6). Some CCSP/VEGF-C-transgenic mice were crossed with Prox1-EGFP FVB/N mice (Mutant Mouse Regional Resource Center, strain 031006-UCD) to create triple-transgenic mice with fluorescent green lymphatics. Mice were genotyped by detection of Prox1-EGFP fluorescence or by PCR analysis of tail DNA using the following primers: CCSP-rtTA forward, 5'-CCTCGATGGTAGACCCGTAA-3', CCSP-rtTA reverse, 5'-CGCAATGGAGCAAAAGTACA-3', tetO-VEGF-C forward, 5'-CCAAACCGGGCCCCTCTGCTAAC-3', tetO-VEGF-C reverse, 5'-ACTGTCCCCTGTCCTGGTATTGAG-3', Prox1-EGFP forward, 5'-ATGTGCCATAAATCCCAGAGCCTAT-3', and Prox1-EGFP reverse, 5'-TAGCGGCTGAAGCACTGCA-3'. Mice housed under pathogen-free conditions were anesthetized for experiments with ketamine (87 $\mathrm{mg} / \mathrm{kg}$, Pfizer, NADA 45-290) and xylazine (10 mg/kg, Bimeda, ANADA 200-529).

\section{Treatments}

According to the standard protocol (Figure 2A), double-transgenic CCSP/VEGF-C mice were weaned at P21 and given doxycycline (Sigma-Aldrich, D9891) $(0.01 \mathrm{mg} / \mathrm{ml})$ in drinking water containing $4 \%$ sucrose, or as otherwise specified, to induce VEGF-C overexpression in respiratory epithelial cells (6). At P28, doxycycline was stopped, and mice were given normal water for 14 days to allow VEGF-C mRNA expression to return to baseline (washout). In initial experiments, maternal mice with newly born litters were given $0.01 \mathrm{or} 0.1 \mathrm{mg} /$ $\mathrm{ml}$ doxycycline in drinking water from $\mathrm{P} 0$ to $\mathrm{P} 7$, and abnormal lymphatic growth was examined thereafter.

Most treatments began at P42 using dosing regimes based on published studies: (a) anti-mouse function-blocking antibodies for VEGFR-2 (DC101) and VEGFR-3 (mF4-31C1) (40 mg/kg i.p. on alternate days, ImClone Systems/Eli Lilly) (6); (b) Notch signaling inhibitor DAPT (N-\{N-(3,5-difluorophenacetyl)-L-alanyl\}-S-phenylglycine t-butyl ester) (1.5 or $10 \mathrm{mg} / \mathrm{kg}$ given 5 days/week by gavage, Calbiochem, 565770); (c) pan $\beta$-adrenergic receptor inhibitor propranolol (5 or $20 \mathrm{mg} / \mathrm{kg}$ i.p. daily, Sigma-Aldrich, P0884); (d) autophagy inhibitor chloroquine (50 mg/kg i.p. daily, Sigma-Aldrich, C6628) (17); (e) antiinflammatory steroid dexamethasone (1 or $10 \mathrm{mg} / \mathrm{kg}$ i.p. daily, Phoenix Pharmaceuticals, ANADA 200-324); and (f) mTOR inhibitor rapamycin (0.5 to $20 \mathrm{mg} / \mathrm{kg}$ i.p. daily, LC Labs, R-5000). Some mice received BrdU (50 mg/kg i.p., Sigma-Aldrich, B9285) to label mitotic cells 3 hours before euthanasia. Controls consisted of single-transgenic mice treated the same as double-transgenic mice or double-transgenic mice that received the active agent vehicle or nothing.

PCR

Perfusion of CCSP/VEGF-C mice with PBS was followed by RNA extraction from tracheas and cDNA synthesis (6). Samples of 5 ng cDNA were analyzed by qRT-PCR using SYBR qRT-PCR SuperMix Universal (Invitrogen) and measured in duplicate with a Bio-Rad MyiQ PCR machine using the following 
protocol: $50^{\circ} \mathrm{C}$ for 2 minutes, $95^{\circ} \mathrm{C}$ for 10 minutes, followed by 40 cycles at $95^{\circ} \mathrm{C}$ for 15 seconds and then at $60^{\circ} \mathrm{C}$ for 60 seconds. Gene expression values were normalized to $\beta$-actin, and results were presented as fold differences in mice treated with doxycycline compared with water controls. Primers (Integrated DNA Technologies) were as follows: VEGF-C forward, 5'-GAAATGTGCCTGTGAATGTACAG-3', VEGF-C reverse, 5'-GACAGTCCTGGATCACAATGC-3', $\beta$-actin forward, 5'-GGCTGTATTCCCCTCCATCG-3', and $\beta$-actin reverse, 5'-CCAGTTGGTAACAATGCCATGT-3'.

Alternatively, tracheas were homogenized in Trizol (Thermo Scientific, catalog 15596026) before isolating RNA. qRT-PCR for VEGFR-3 and $\beta$-actin was performed using the SensiFAST SYBR Hi-ROX One-Step Kit (Bioline USA Inc., BIO-73005) using primers with the following sequences: VEGFR-3, GCTGTTGGTTGGAGAGAAGC/TGCTGGAGAGTTCTGTGTGG, and, $\beta$-actin, GCTCTTTTCCAGCCTTCCTT/CTTCTGCATCCTGTCAGCAA.

qRT-PCR for Prox1 was performed using the TaqMan EZ RT-PCR Kit (ABI, N8080236) using primers with the following sequences: Prox1, AGTTCCACAGACCAGACGGAA/FAM-CCCTTCCCCTGGTGGTCCGAA-BKH1/GCAGATTGCTCGGATGAGTTT, and Gapdh, TCACTGGCATGGCCTTCC/ JOE-TTCCTACCCCCAATGTGTCCGTCG-TAMRA/GGCGGCACGTCAGATCC.

\section{Immunohistochemistry}

Anesthetized mice were perfused for 2 minutes with fixative (1\% paraformaldehyde in PBS, pH 7.4) via a cannula inserted through the left ventricle into the aorta. Tissues were removed and immersed in fixative for 1 hour at room temperature. Tissues were washed with PBS and stained immunohistochemically by incubating whole mounts with one or more of primary antibody (Supplemental Table 1) diluted in PBS containing $0.3 \%$ Triton X-100, $0.2 \%$ bovine serum albumin, $5 \%$ normal donkey or goat serum, and $0.1 \%$ sodium azide (6). A rat antibody to BrdU was used to detect dividing cells using acid hydrolysis and alkali neutralization procedures before the antibody-staining step (84). The specificity of antibodies to p-mTOR, p-S6RP, and p-4E-BP1 was confirmed by preabsorption with blocking peptides that abolished staining with primary antibodies (Supplemental Figure $4 \mathrm{H}$ ). Fluorescent secondary antibodies were labeled with Cy3, Alexa Fluor 488, or 647 (Jackson ImmunoResearch).

\section{Microscopy and measurements}

Measurement of lymphangiectasia severity. The extent of lymphangiectasia was assessed by line density measurements of LYVE-1-stained lymphatics in tracheal whole mounts. Four 1,024 $\times 1,024$ pixel images, each showing lymphatics in two intercartilage regions measuring 1,300 × 1,300 $\mu \mathrm{m}$ (Figure $2 \mathrm{~B}$ ), were obtained from each trachea by confocal microscopy (Zeiss LSM510, objective $\times 10$; zoom 0.7 and AIM4.2 software). After importing the image into Image ( NIH, version $1.48 \mathrm{v}$, http://imagej.nih.gov/ij), 3 equidistant parallel survey lines were drawn perpendicular to the lymphatics (Figure 2B), and the fluorescence intensities of pixels along the lines were recorded. The line density of lymphatics was calculated as the proportion of LYVE-1 pixels with fluorescence intensity above an empirically set threshold of 100 on a scale of 0 to 255 , which distinguished lymphatics from background fluorescence. The mean value for experimental groups was calculated from the mean line density for all images of each mouse in the group ( $n=$ number of mice/group).

Measurement of cell number. For data in Figure 4H, Prox1-positive nuclei were counted automatically using the ImageJ Tool for Counting Nuclei plug-in (https://imagej.nih.gov/ij/plugins/itcn.html) on confocal microscopic images of Prox1-stained tracheal whole mounts. Two $\times 10$ images per trachea were converted to grayscale and inverted with ImageJ. Counts were made with the following parameters: threshold, 8 (on a scale of 0 to 255); nuclear width, 7 pixels; minimum distance between nuclei, 5 pixels.

Other cell counts were made directly on tracheal whole mounts stained for BrdU, Prox1, activated caspase-3, or p-4E-BP1 viewed in real-time with an Olympus DP73 color CCD camera on a Zeiss Axiophot fluorescence microscope. Using custom software, fluorescent cells of interest were counted manually on projected images on a computer monitor, marked by color-coded dots, and the number of cells was expressed per area of tissue. Discontinuities in luminal staining by podocalyxin in LYVE-1-stained lymphatics were counted and expressed as the number of breaks per trachea.

Measurement of fluorescence intensity. The intensities of Prox1 and VEGFR-3 immunofluorescence in tracheal lymphatics from mice, treated with vehicle or rapamycin, were compared in $\times 10$ confocal microscopic images made with identical acquisition parameters. The images were imported into ImageJ, and 
intensity histograms were prepared. The fluorescence intensity of each image was calculated from the percentage of pixels above an empirically determined threshold of 80 . Values for rapamycin-treated mice were normalized to those for vehicle-treated mice. Surface plots were prepared with color-coded lookup tables on ImageJ.

\section{Measurement of rapamycin blood concentration and systemic effects}

Tail blood $(200 \mu \mathrm{l})$ from mice that received rapamycin for 14 days $(0.5-20 \mathrm{mg} / \mathrm{kg}$ i.p. daily) was collected into EDTA tubes at 1 hour and again at 24 hours after the final dose. Tracheas from the same mice were prepared for immunohistochemical staining. Rapamycin concentrations in whole blood were measured by HPLC/mass spectrometry as described previously (85). The half-life of rapamycin in blood was calculated assuming an exponential rate of clearance. Blood cell counts were made with a Hemavet 950 veterinary cytometer (Drew Scientific). Mice were weighed daily. Thymus weight was expressed as a percentage of body weight.

\section{Statistics}

Four to five mice per group were used to generate data unless otherwise indicated. Bar graphs show mean \pm SEM. Data were analyzed and graphs were prepared with Prism 6.0 (GraphPad Software). Differences between multiple groups were assessed by ANOVA followed by the Dunn-Bonferroni test. Differences between frequency distributions of nonnormally distributed data, such as immunoreactivity intensities, were assessed by the Kolmogorov-Smirnov 2-sample test. $P$ values of less than 0.05 were considered statistically significant.

\section{Study approval}

All experimental procedures were approved by the Institutional Animal Care and Use Committee of the University of California, San Francisco.

\section{Author contributions}

PB, LCY, JCF, YKH, and DMD designed studies. PB, LCY, JCF, DC, and YKH performed experiments and acquired and analyzed data. PB, LCY, JCF, and DMD wrote the manuscript.

\section{Acknowledgments}

This work was supported by generous support from the Lymphatic Malformation Institute; grants to DMD from the NIH National Heart, Lung, and Blood Institute (R01 HL127402, R01 HL059157, and P01 HL024136), the Leducq Foundation (11CVD03), and Angel-Works Foundation and by funding to YKH from the National Heart, Lung, and Blood Institute (HL121036 and HL119583). We thank Michael Dellinger of the University of Texas, Southwestern Medical Center, Dallas, for many insightful suggestions; Kari Alitalo of the University of Helsinki for the tetO-mVEGF-C responder mice; Martin A. Javors of the University of Texas, San Antonio, for measuring rapamycin in blood; and Bronek Pytowski of Eli Lilly for function-blocking antibodies to mouse VEGFR-2 and VEGFR-3.

Address correspondence to: Peter Baluk, University of California, San Francisco, 513 Parnassus Avenue, San Francisco, California 94143, USA. Phone: 415.476.2118; Email: peter.baluk@ucsf.edu.

LCY's present address is: The Jackson Laboratory, Sacramento, California, USA.

\footnotetext{
1. Wassef M, et al. Vascular anomalies classification: recommendations from the International Society for the Study of Vascular Anomalies. Pediatrics. 2015;136(1):e203-e214.

2. Brouillard P, Boon L, Vikkula M. Genetics of lymphatic anomalies. J Clin Invest. 2014;124(3):898-904.

3. Trenor CC, Chaudry G. Complex lymphatic anomalies. Semin Pediatr Surg. 2014;23(4):186-190.

4. Adams DM, et al. Efficacy and safety of sirolimus in the treatment of complicated vascular anomalies. Pediatrics. 2016;137(2):e20153257.

5. Virchow R. Gesammelte Abhandlungen zur wissenschaftlichen Medicin. Frankfurt-am-Main, Germany: Verlag Meidinger Sohn \& Comp.; 1856;983. https://archive.org/details/b21462161. Accessed July 18, 2017

6. Yao LC, et al. Pulmonary lymphangiectasia resulting from vascular endothelial growth factor-C overexpression during a critical period. Circ Res. 2014;114(5):806-822.
} 
7. Yuan SM. Congenital pulmonary lymphangiectasia [published online ahead of print July 18, 2017]. J Perinat Med. https://doi. org/10.1515/jpm-2016-0407.

8. Myers A, et al. Perinatal features of the RASopathies: Noonan syndrome, cardiofaciocutaneous syndrome and Costello syndrome. Am J Med Genet A. 2014;164A(11):2814-2821.

9. Limaye N, et al. Somatic activating PIK3CA mutations cause venous malformation. Am J Hum Genet. 2015;97(6):914-921.

10. Luks VL, et al. Lymphatic and other vascular malformative/overgrowth disorders are caused by somatic mutations in PIK $3 \mathrm{CA}$. J Pediatr. 2015;166(4):1048-54.e1.

11. Wang Z, Li K, Yao W, Dong K, Xiao X, Zheng S. Successful treatment of kaposiform lymphangiomatosis with sirolimus. Pediatr Blood Cancer. 2015;62(7):1291-1293.

12. Rössler J, Geiger J, Földi E, Adams DM, Niemeyer CM. Sirolimus is highly effective for lymph leakage in microcystic lymphatic malformations with skin involvement. Int J Dermatol. 2017;56(4):e72-e75.

13. Yao LC, Baluk P, Feng J, McDonald DM. Steroid-resistant lymphatic remodeling in chronically inflamed mouse airways. Am J Pathol. 2010;176(3):1525-1541.

14. Larrivée B, et al. ALK1 signaling inhibits angiogenesis by cooperating with the Notch pathway. Dev Cell. 2012;22(3):489-500.

15. Stiles JM, et al. Targeting of beta adrenergic receptors results in therapeutic efficacy against models of hemangioendothelioma and angiosarcoma. PLOS ONE. 2013;8(3):e60021.

16. Poralla C, Specht S, Born M, Müller A, Bartmann P, Müller A. Treatment of congenital generalized lymphangiectasia with propranolol in a preterm infant. Pediatrics. 2014;133(2):e439-e442.

17. Parkhitko A, et al. Tumorigenesis in tuberous sclerosis complex is autophagy and p62/sequestosome 1 (SQSTM1)-dependent Proc Natl Acad Sci USA. 2011;108(30):12455-12460.

18. Taveira-DaSilva AM, Moss J. Clinical features, epidemiology, and therapy of lymphangioleiomyomatosis. Clin Epidemiol. 2015;7:249-257.

19. Hos D, et al. Suppression of inflammatory corneal lymphangiogenesis by application of topical corticosteroids. Arch Ophthalmol. 2011;129(4):445-452.

20. Boscolo E, et al. AKT hyper-phosphorylation associated with PI3K mutations in lymphatic endothelial cells from a patient with lymphatic malformation. Angiogenesis. 2015;18(2):151-162.

21. Cao L, Arany PR, Wang YS, Mooney DJ. Promoting angiogenesis via manipulation of VEGF responsiveness with notch signaling. Biomaterials. 2009;30(25):4085-4093.

22. Hernandez SL, et al. Notch and VEGF pathways play distinct but complementary roles in tumor angiogenesis. Vasc Cell. 2013;5(1):17.

23. Léauté-Labrèze C, Dumas de la Roque E, Hubiche T, Boralevi F, Thambo JB, Taïeb A. Propranolol for severe hemangiomas of infancy. NEngl J Med. 2008;358(24):2649-2651.

24. Ozeki M, Fukao T, Kondo N. Propranolol for intractable diffuse lymphangiomatosis. N Engl J Med. 2011;364(14):1380-1382.

25. Greenberger S, Bischoff J. Infantile hemangioma-mechanism(s) of drug action on a vascular tumor. Cold Spring Harb Perspect Med. 2011;1(1):a006460.

26. Parkhitko AA, et al. Autophagy-dependent metabolic reprogramming sensitizes TSC2-deficient cells to the antimetabolite 6-aminonicotinamide. Mol Cancer Res. 2014;12(1):48-57.

27. McCormack FX, et al. Efficacy and safety of sirolimus in lymphangioleiomyomatosis. N Engl J Med. 2011;364(17):1595-1606.

28. Goldberg HJ, et al. Everolimus for the treatment of lymphangioleiomyomatosis: a phase II study. Eur Respir J. 2015;46(3):783-794.

29. Eng CP, Sehgal SN, Vézina C. Activity of rapamycin (AY-22,989) against transplanted tumors. J Antibiot. 1984;37(10):1231-1237.

30. Novalic Z, et al. Dose-dependent effects of sirolimus on mTOR signaling and polycystic kidney disease. $J$ Am Soc Nephrol. 2012;23(5):842-853

31. Fabian MC, Lakey JR, Rajotte RV, Kneteman NM. The efficacy and toxicity of rapamycin in murine islet transplantation. In vitro and in vivo studies. Transplantation. 1993;56(5):1137-1142.

32. Luo H, Duguid W, Chen H, Maheu M, Wu J. The effect of rapamycin on T cell development in mice. Eur J Immunol. 1994;24(3):692-701.

33. Meier-Kriesche HU, Kaplan B. Toxicity and efficacy of sirolimus: relationship to whole-blood concentrations. Clin Ther. 2000;22 Suppl B:B93-100.

34. Laplante M, Sabatini DM. mTOR signaling in growth control and disease. Cell. 2012;149(2):274-293.

35. Li J, Kim SG, Blenis J. Rapamycin: one drug, many effects. Cell Metab. 2014;19(3):373-379.

36. Flores MV, Hall CJ, Crosier KE, Crosier PS. Visualization of embryonic lymphangiogenesis advances the use of the zebrafish model for research in cancer and lymphatic pathologies. Dev Dyn. 2010;239(7):2128-2135.

37. Hoopes SL, Willcockson HH, Caron KM. Characteristics of multi-organ lymphangiectasia resulting from temporal deletion of calcitonin receptor-like receptor in adult mice. PLOS ONE. 2012;7(9):e45261.

38. Zheng W, Aspelund A, Alitalo K. Lymphangiogenic factors, mechanisms, and applications. J Clin Invest. 2014;124(3):878-887.

39. Wu JK, et al. Aberrant lymphatic endothelial progenitors in lymphatic malformation development. PLoS One. 2015;10(2):e0117352.

40. Davis RB, Kechele DO, Blakeney ES, Pawlak JB, Caron KM. Lymphatic deletion of calcitonin receptor-like receptor exacerbates intestinal inflammation. JCI Insight. 2017;2(6):e92465.

41. Osborn AJ, et al. Activating PIK3CA alleles and lymphangiogenic phenotype of lymphatic endothelial cells isolated from lymphatic malformations. Hum Mol Genet. 2015;24(4):926-938.

42. Itakura E, Yamamoto H, Oda Y, Furue M, Tsuneyoshi M. VEGF-C and VEGFR-3 in a series of lymphangiomas: is superficial lymphangioma a true lymphangioma? Virchows Arch. 2009;454(3):317-325.

43. Partanen TA, et al. Neuropilin-2 and vascular endothelial growth factor receptor-3 are up-regulated in human vascular malformations. Angiogenesis. 2013;16(1):137-146.

44. Pullinger BD, Florey HW. Proliferation of lymphatics in inflammation. J Pathol Bact. 1937;45:157-170.

45. Lohela M, Heloterä H, Haiko P, Dumont DJ, Alitalo K. Transgenic induction of vascular endothelial growth factor-C is strongly 
angiogenic in mouse embryos but leads to persistent lymphatic hyperplasia in adult tissues. Am J Pathol. 2008;173(6):1891-1901.

46. Nurmi H, Saharinen P, Zarkada G, Zheng W, Robciuc MR, Alitalo K. VEGF-C is required for intestinal lymphatic vessel maintenance and lipid absorption. EMBO Mol Med. 2015;7(11):1418-1425.

47. Zarkada G, Heinolainen K, Makinen T, Kubota Y, Alitalo K. VEGFR3 does not sustain retinal angiogenesis without VEGFR2. Proc Natl Acad Sci USA. 2015;112(3):761-766.

48. Cursiefen C, Maruyama K, Jackson DG, Streilein JW, Kruse FE. Time course of angiogenesis and lymphangiogenesis after brief corneal inflammation. Cornea. 2006;25(4):443-447.

49. Betterman KL, et al. Remodeling of the lymphatic vasculature during mouse mammary gland morphogenesis is mediated via epithelial-derived lymphangiogenic stimuli. Am J Pathol. 2012;181(6):2225-2238.

50. Mumprecht V, Roudnicky F, Detmar M. Inflammation-induced lymph node lymphangiogenesis is reversible. Am J Pathol. 2012;180(3):874-879.

51. Lammoglia GM, Van Zandt CE, Galvan DX, Orozco JL, Dellinger MT, Rutkowski JM. Hyperplasia, de novo lymphangiogenesis, and lymphatic regression in mice with tissue-specific, inducible overexpression of murine VEGF-D. Am J Physiol Heart Circ Physiol. 2016;311(2):H384-H394.

52. Karpanen T, et al. Lymphangiogenic growth factor responsiveness is modulated by postnatal lymphatic vessel maturation. $A m J$ Pathol. 2006;169(2):708-718.

53. Léauté-Labrèze $\mathrm{C}$, et al. A randomized, controlled trial of oral propranolol in infantile hemangioma. N Engl J Med. 2015;372(8):735-746.

54. Ozeki M, et al. Propranolol as an alternative treatment option for pediatric lymphatic malformation. Tohoku J Exp Med. 2013;229(1):61-66.

55. Baud J, Lomri A, Graber D, Bikfalvi A. The therapeutic response in Gorham's syndrome to the beta-blocking agent propranolol is correlated to VEGF-A, but not to VEGF-C or FLT1 expression. BMC Res Notes. 2015;8:333.

56. Geudens I, et al. Role of delta-like-4/Notch in the formation and wiring of the lymphatic network in zebrafish. Arterioscler Thromb Vasc Biol. 2010;30(9):1695-1702.

57. Murtomaki A, et al. Notch1 functions as a negative regulator of lymphatic endothelial cell differentiation in the venous endothelium. Development. 2013;140(11):2365-2376.

58. Fatima A, et al. Murine Notch1 is required for lymphatic vascular morphogenesis during development. Dev Dyn. 2014;243(7):957-964.

59. Zheng W, et al. Notch restricts lymphatic vessel sprouting induced by vascular endothelial growth factor. Blood. 2011;118(4):1154-1162.

60. Niessen K, et al. The Notch1-D114 signaling pathway regulates mouse postnatal lymphatic development. Blood. 2011;118(7):1989-1997.

61. Henske EP, McCormack FX. Lymphangioleiomyomatosis - a wolf in sheep's clothing. J Clin Invest. 2012;122(11):3807-3816.

62. Taveira-DaSilva AM, Hathaway O, Stylianou M, Moss J. Changes in lung function and chylous effusions in patients with lymphangioleiomyomatosis treated with sirolimus. Ann Intern Med. 2011;154(12):797-805.

63. Huber S, et al. Inhibition of the mammalian target of rapamycin impedes lymphangiogenesis. Kidney Int. 2007;71(8):771-777.

64. Kobayashi S, Kishimoto T, Kamata S, Otsuka M, Miyazaki M, Ishikura H. Rapamycin, a specific inhibitor of the mammalian target of rapamycin, suppresses lymphangiogenesis and lymphatic metastasis. Cancer Sci. 2007;98(5):726-733.

65. Luo Y, et al. Rapamycin inhibits lymphatic endothelial cell tube formation by downregulating vascular endothelial growth factor receptor 3 protein expression. Neoplasia. 2012;14(3):228-237.

66. Mäkinen T, et al. Isolated lymphatic endothelial cells transduce growth, survival and migratory signals via the VEGF-C/D receptor VEGFR-3. EMBO J. 2001;20(17):4762-4773.

67. Dieterich LC, et al. DeepCAGE transcriptomics reveal an important role of the transcription factor MAFB in the lymphatic endothelium. Cell Rep. 2015;13(7):1493-1504.

68. Gupta S, et al. Binding of ras to phosphoinositide 3-kinase p110alpha is required for ras-driven tumorigenesis in mice. Cell. 2007;129(5):957-968.

69. Zhou F, et al. Akt/Protein kinase B is required for lymphatic network formation, remodeling, and valve development. Am $J$ Pathol. 2010;177(4):2124-2133.

70. Mouta-Bellum C, et al. Organ-specific lymphangiectasia, arrested lymphatic sprouting, and maturation defects resulting from gene-targeting of the PI3K regulatory isoforms p85alpha, p55alpha, and p50alpha. Dev Dyn. 2009;238(10):2670-2679.

71. Koch S, Tugues S, Li X, Gualandi L, Claesson-Welsh L. Signal transduction by vascular endothelial growth factor receptors Biochem J. 2011;437(2):169-183.

72. Deng Y, Atri D, Eichmann A, Simons M. Endothelial ERK signaling controls lymphatic fate specification. J Clin Invest. 2013;123(3):1202-1215.

73. Lei FR, Li XQ, Liu H, Zhu RD, Meng QY, Rong JJ. Rapamycin and 3-methyladenine regulate apoptosis and autophagy in bone-derived endothelial progenitor cells. Chin Med J. 2012;125(22):4076-4082.

74. Baraz R, et al. mTOR inhibition by everolimus in childhood acute lymphoblastic leukemia induces caspase-independent cell death. PLoS ONE. 2014;9(7):e102494.

75. Ekshyyan O, et al. Anti-lymphangiogenic properties of mTOR inhibitors in head and neck squamous cell carcinoma experimental models. BMC Cancer. 2013;13:320.

76. Kwon S, et al. Rapamycin up-regulates triglycerides in hepatocytes by down-regulating Prox1. Lipids Health Dis. $2016 ; 15: 41$.

77. Johnson NC, et al. Lymphatic endothelial cell identity is reversible and its maintenance requires Prox1 activity. Genes Dev. 2008;22(23):3282-3291.

78. Srinivasan RS, et al. The Prox1-Vegfr3 feedback loop maintains the identity and the number of lymphatic endothelial cell progenitors. Genes Dev. 2014;28(19):2175-2187.

79. Choo AY, Yoon SO, Kim SG, Roux PP, Blenis J. Rapamycin differentially inhibits S6Ks and 4E-BP1 to mediate cell-type-specific repression of mRNA translation. Proc Natl Acad Sci USA. 2008;105(45):17414-17419.

80. Hammill AM, et al. Sirolimus for the treatment of complicated vascular anomalies in children. Pediatr Blood Cancer. 
2011;57(6):1018-1024.

81. Lackner H, et al. Sirolimus for the treatment of children with various complicated vascular anomalies. Eur J Pediatr. 2015;174(12):1579-1584.

82. Mori M, et al. Pulmonary and pleural lymphatic endothelial cells from pediatric, but not adult, patients with Gorham-Stout disease and generalized lymphatic anomaly, show a high proliferation rate. Orphanet J Rare Dis. 2016;11(1):67.

83. Yao J, Taveira-DaSilva AM, Jones AM, Julien-Williams P, Stylianou M, Moss J. Sustained effects of sirolimus on lung function and cystic lung lesions in lymphangioleiomyomatosis. Am J Respir Crit Care Med. 2014;190(11):1273-1282.

84. Jackson D, Cook PR. Analyzing DNA replication III: antibody labeling of incorporated bromodeoxyuridine (BrdU) in tissues and cells. CSH Protoc. 2008;2008:pdb.prot5033.

85. Zhang Y, et al. Rapamycin extends life and health in C57BL/6 mice. J Gerontol A Biol Sci Med Sci. 2014;69(2):119-130. 